\title{
Imaging methods to evaluate tumor microenvironment factors affecting nanoparticle drug delivery and antitumor response
}

\author{
Amber S. Moody ${ }^{1,2,3,4}$, Paul A. Dayton ${ }^{1,2,4}$, William C. Zamboni $i^{1,2,3}$ \\ 'UNC Eshelman School of Pharmacy, University of North Carolina, Chapel Hill, NC 27599, USA. \\ ¿UNC Lineberger Comprehensive Cancer Center, Chapel Hill, NC 27599, USA. \\ ${ }^{3}$ Carolina Institute for Nanomedicine, Chapel Hill, NC 27599, USA. \\ ${ }^{4}$ Joint Department of Biomedical Engineering, University of North Carolina and North Carolina State University, Chapel Hill, NC \\ 27599, USA.
}

Correspondence to: Dr. William C. Zamboni, UNC Eshelman School of Pharmacy, University of North Carolina, Mason Farm Road, Chapel Hill, NC 27599, USA. E-mail: zamboni@email.unc.edu

How to cite this article: Moody AS, Dayton PA, Zamboni WC. Imaging methods to evaluate tumor microenvironment factors affecting nanoparticle drug delivery and antitumor response. Cancer Drug Resist 2021;4:382-413.

http://dx.doi.org/10.20517/cdr.2020.94

Received: 15 Oct 2020 First Decision: 14 Dec 2020 Revised: 7 Jan 2021 Accepted: 28 Jan 2021 Available online: 19 Jun 2021

Academic Editor: Godefridus J. Peters Copy Editor: Yue-Yue Zhang Production Editor: Xi-Jun Chen

\begin{abstract}
Standard small molecule and nanoparticulate chemotherapies are used for cancer treatment; however, their effectiveness remains highly variable. One reason for this variable response is hypothesized to be due to nonspecific drug distribution and heterogeneity of the tumor microenvironment, which affect tumor delivery of the agents. Nanoparticle drugs have many theoretical advantages, but due to variability in tumor microenvironment (TME) factors, the overall drug delivery to tumors and associated antitumor response are low. The nanotechnology field would greatly benefit from a thorough analysis of the TME factors that create these physiological barriers to tumor delivery and treatment in preclinical models and in patients. Thus, there is a need to develop methods that can be used to reveal the content of the TME, determine how these TME factors affect drug delivery, and modulate TME factors to increase the tumor delivery and efficacy of nanoparticles. In this review, we will discuss TME factors involved in drug delivery, and how biomedical imaging tools can be used to evaluate tumor barriers and predict drug delivery to tumors and antitumor response.
\end{abstract}

Keywords: Tumor microenvironment, imaging, drug delivery, nanoparticles

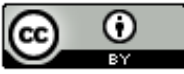

(C) The Author(s) 2021. Open Access This article is licensed under a Creative Commons Attribution 4.0 International License (https://creativecommons.org/licenses/by/4.0/), which permits unrestricted use, sharing, adaptation, distribution and reproduction in any medium or format, for any purpose, even commercially, as long as you give appropriate credit to the original author(s) and the source, provide a link to the Creative Commons license, and indicate if changes were made.

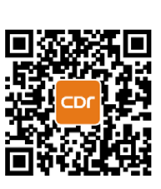




\section{INTRODUCTION}

The tumor microenvironment (TME) is characterized by many factors including increased concentrations of extracellular matrix proteins such as collagen, hyaluronan, and fibronectins, as well as low extracellular $\mathrm{pH}$, tortuous neovasculature, and hypoxia ${ }^{[1]}$. This environment plays an essential role in tumor development and response to therapy, and is often highly heterogeneous even within the same tumor type and the same patient ${ }^{[1]}$. The gold standard of cancer treatment is chemotherapy; however, the effectiveness is limited due to heterogeneity of the TME itself, multidrug resistance, and nonspecific drug distribution ${ }^{[2]}$. The likelihood of a chemotherapeutic crossing tumor vasculature to act on cancer cells is inherently decreased due to physical barriers created based on these varying factors in the TME. To overcome these barriers, there has been an increasing trend toward utilizing nanotechnology. Nanotechnology has many theoretical advantages in regards to cancer treatment including prolonged exposure, increased solubility, targeted delivery, and overall improved therapeutic index ${ }^{[3]}$. There is also an expected increase in permeability when using nanotechnology due to their nanometer size and the association with the enhanced permeability and retention (EPR) effect. In theory, the EPR effect is caused by leaky neovasculature and defective lymphatic drainage ${ }^{[4,5]}$. However, the factors that affect EPR and the true presence of the EPR effects in preclinical tumor models and especially in patients with solid tumors are unclear. In addition, it is possible that the EPR effect is highly variable across tumor types and within patients.

A few nanoparticle agents have been developed and approved by the United States Food and Drug Administration (FDA) for the treatment of solid tumors including PEGylated liposomal doxorubicin $\left(\right.$ Doxil $\left.^{\circ}\right)$, liposomal daunorubicin (DaunoXome ${ }^{\odot}$ ), liposomal irinotecan $\left(\right.$ Onivyde $\left.^{\odot}\right)$, and paclitaxel albuminbound particles $\left(\mathrm{Abraxane}^{\infty}\right)^{[6,7]}$. Although treatment response has improved with the development of these nanoparticle drugs, in many cases the theoretical advantages of these drugs are not fully realized. The lower than expected efficacy of nanoparticles may be a result of the overall low tumor uptake of these agents that is due to the heterogeneity of the components that comprise the $\mathrm{TME}^{[4,5]}$. The varying factors of the TME that contribute to this heterogeneity include angiogenesis, stiff extracellular matrix, high interstitial fluid pressures, hypoxia, acidic $\mathrm{pH}$, and the presence of immune cells (e.g., macrophages). A characteristic of tumor growth is angiogenesis where the rapid growing neovasculature are incredibly fragile, tortuous or containing abnormal twists and turns, irregularly shaped, dilated, and highly permeable with increased geometric resistance ${ }^{[8]}$. This abnormal vascular network results in heterogeneous hypoperfused or necrotic areas within tumor tissue ${ }^{[9]}$ with variability in blood distribution leading to variability in drug distribution ${ }^{[4]}$. After extravasation of the nanotherapy agent through the tumor vasculature, the stiff extracellular matrix caused by activated cancer associated fibroblasts and upregulation of extracellular matrix proteins exists as a barrier to the diffusional movement of the agent through the interstitial space ${ }^{[10]}$. High interstitial fluid pressures also prevent extravasation of the nanotherapy agents where they are expelled into systemic circulation from the tumor periphery into adjoining tissues ${ }^{[11]}$. Hypoxia further prevents nanotherapy from reaching various parts of the tumor by causing irregular vasculature and poorly perfused regions ${ }^{[12]}$. Immune cells, such as macrophages and monocytes, have been shown to take up nanoparticles in circulation, tumor, and tissues, and also alter the delivery of nanoparticles to tumors ${ }^{[13,14]}$.

Many combinational therapies have been developed to improve the efficacy of nanoparticles; however, there are still several identified and unidentified factors and barriers in the TME that inhibit nanoparticle delivery to tumors ${ }^{[15]}$. The most promising approach to improving drug delivery and efficacy in solid tumors is to modify or "normalize" the TME. This also demonstrates the need for methods to evaluate the components of the TME. These methods may also allow for greater efficacy of nanoparticle agents and potentially personalized treatment methods for patients or specific tumors and monitoring of treatment response ${ }^{[16]}$. Table 1 summarizes the factors within the TME, their effect on nanoparticle delivery to solid tumors, and the imaging methods that can be used to measure these TME factors. The response of the TME to selected treatment methods is also an important factor to consider in determining continuation of 
Table 1. Summary of tumor microenvironment factors, their effect on drug delivery to tumors, and characterization methods for each factor

\begin{tabular}{|c|c|c|}
\hline TME factor & Effect on tumor drug delivery & Characterization method \\
\hline $\begin{array}{l}\text { Extracellular matrix proteins: } \\
\text { collagen, proteoglycan } \\
\text { (hyaluronan), fibronectin, and } \\
\text { lamanin }\end{array}$ & $\begin{array}{l}\text { Contribute to tissue density and stiffness, and creating } \\
\text { a physical barrier to drug penetration }\end{array}$ & $\mathrm{OCT}, \mathrm{PAI}$, and $\mathrm{MRI}^{\star}$ \\
\hline Matrix metalloproteinase & $\begin{array}{l}\text { Upregulates anti-apoptotic molecules protecting cancer } \\
\text { cells from chemo-induced apoptosis }{ }^{[18]}\end{array}$ & $\begin{array}{l}\text { Optical imaging (fluorescence, } \\
\text { bioluminescence), PET, SPECT, MRI, PAI, and } \\
\text { FMT }^{\star}\end{array}$ \\
\hline $\begin{array}{l}\text { Mesenchymal stromal (Stem) } \\
\text { cells }\end{array}$ & $\begin{array}{l}\text { Can differentiate into various cell types and protect } \\
\text { cancer cells from external aggression allowing them to } \\
\text { escape apoptosis }{ }^{[19]}\end{array}$ & $\begin{array}{l}\text { Fluorescence, bioluminescence, MRI, PET, and } \\
\text { SPECT }\end{array}$ \\
\hline Cancer associated fibroblasts & $\begin{array}{l}\text { Produce extracellular matrix proteins creating a physical } \\
\text { barrier to drug delivery }{ }^{[20,21]}\end{array}$ & MRI \\
\hline Immune cells & $\begin{array}{l}\text { Down regulates proapoptotic molecules and } \\
\text { upregulates interleukin- } 6 \text {, protecting tumor cells from } \\
\text { chemo-induced cell death }{ }^{[22]}\end{array}$ & $\begin{array}{l}\text { MRI, PAI, fluorescence, ultrasound, } \mathrm{CT}^{\star}, \mathrm{PET} \text {, } \\
\text { SPECT, and bioluminescence }\end{array}$ \\
\hline $\begin{array}{l}\text { Tumor vasculature and } \\
\text { lymphatics }\end{array}$ & $\begin{array}{l}\text { Upregulates VEGF, modifying the apoptotic signaling } \\
\text { pathway and expressing anti-apoptotic proteins }{ }^{[23]} \text { and } \\
\text { has varied and disorganized blood flow }{ }^{[24]} \text { leading to } \\
\text { variability in drug distribution }\end{array}$ & $\begin{array}{l}\text { MRI, CT, PET, ultrasound, PAI, intravital } \\
\text { microscopy, OCT, fluorescence, and } \\
\text { bioluminescence }\end{array}$ \\
\hline $\begin{array}{l}\text { Metabolic-choline- } \\
\text { phospholipid metabolism }\end{array}$ & $\begin{array}{l}\text { Increases levels of glutathione, reducing ROH species } \\
\text { resulting in chemoresistance }\end{array}$ & MRI and PET \\
\hline Hypoxia & $\begin{array}{l}\text { Induces radio- and chemo-resistance through } \\
\text { the mechanism of reactive oxygen radicals that } \\
\text { damage DNA and down-regulate expession of DNA } \\
\text { topoisomerase } I^{[26]}\end{array}$ & PET, MRI, and CT \\
\hline Glycolysis & $\begin{array}{l}\text { Leads to increased production of lactic acid and } \mathrm{H}+ \\
\text { inducing hypoxia and reduced } \mathrm{pH}^{[27]}\end{array}$ & PET \\
\hline $\mathrm{pH}$ & $\begin{array}{l}\text { Influences drug uptake based on the acidity of the } \\
\text { drug, protonating basic chemotherapeutics or allowing } \\
\text { accumulation of acidic chemotherapeutics }\end{array}$ & $\begin{array}{l}\mathrm{CT}, \mathrm{MRI}, \mathrm{PET} \text {, fluorescence, bioluminescence, } \\
\text { and PAI }\end{array}$ \\
\hline Tumor-stroma Interactions & $\begin{array}{l}\text { Abnormal context of the TME facilitates abnormal } \\
\text { cross-talk allowing tumor cells to disregard rules and } \\
\text { adapt to the multicellular environment }\end{array}$ & $\begin{array}{l}\text { Fluorescence, intravital microscopy, MRI, and } \\
\text { PET }\end{array}$ \\
\hline
\end{tabular}

*MRI: Magnetic resonance imaging; PET: positron emission tomography; SPECT: single photon emission computed tomography; CT: computed tomography; PAI: photoacoustic imaging; FMT: fluorescence molecular tomography; OCT: optical coherence tomography.

treatment; however, this topic is beyond the scope of this review. In this review we will summarize current imaging methods used to identify TME factors that could affect nano-drug delivery in an effort to provide insight on evaluating and modulating these factors as ways to improve nanoparticle drug delivery and efficacy.

\section{IMAGING THE TUMOR MICROENVIRONMENT}

Many cancer therapies can be more or less effective depending on the components of the TME. For example, the primary mechanism of radiation therapy is the generation of reactive oxygen species where hypoxic tumors are radiation resistant. Hypoxia and other extracellular matrix proteins including collagen also act as a barrier to some chemotherapy, resulting in further disease progression ${ }^{[2]}$. To evaluate the mechanism of resistance of the TME to therapy, nanoparticles have been engineered to be stimuliresponsive when they interact with changes in physiochemical parameters such as changes in temperature, light, reduction/oxidation enzymes, or $\mathrm{pH}$. These drugs include functional computed tomography (CT) contrast nanoagents that respond to the acidic $\mathrm{pH}$ in the tumor microenvironment ${ }^{[30]}$ and glutathione ${ }^{[31]}$, and fluorescent nanoprobes utilizing quantum dots that are "switched on" when binding to matrix metalloproteinase ${ }^{[32]}$. The EPR effect in the TME has also been evaluated where tumor accumulation of fermuxytol iron nanoparticles imaged with magnetic resonance imaging (MRI) is correlated to tumor delivery of nanoliposomal irinotecan ${ }^{[33]}$. Other nanoparticles used in imaging can be targeted with tumor specific antigens to allow for monitoring of cancer nanotherapy including tumor associated macrophage 
targeted fluorescent nanoparticles (ferumoxytol-VT680XL) ${ }^{[34]}$, antigen specific targeted nanoparticles radiolabeled with indium-111 and imaged with single photon emission computed tomography (SPECT $)^{[35]}$, positron emission tomography (PET) imaged ${ }^{64} \mathrm{Cu}$-labeled HER-2 targeted PEGylated liposomal doxorubicin for HER-2 positive metastatic breast cancer $^{[36]}$, and targeted ultrasound contrast agents (or targeted microbubbles) for vascular endothelial growth factor receptor-2 or $\mathrm{a}_{\mathrm{v}} \mathrm{b}_{3}$ used to image tumor neovasculature and aid in assessment of tumor malignancy and treatment response ${ }^{[37]}$. This allows the nanoparticle agents to be used for targeted delivery and interaction with the TME, resulting in increased accumulation, facilitated drug release, and an increase in uniform distribution ${ }^{[38]}$. This type of active targeting depends on the specific interaction of the designed nanoparticle with the targeted component of the TME. Tracking of cells that interact with the TME is also useful in determining their use for antitumor therapy where mesenchymal stem cells can be tracked using organic semiconducting polymer nanoparticles imaged with photoacoustic imaging ${ }^{[39]}$ and superparamagnetic iron oxide nanoparticles with MRI ${ }^{[40]}$. Targeted delivery and tracking facilitate the need for a method to easily determine if the targeted or altered conditions are present in the TME and monitor the changes in these factors during and post treatment to determine the effectiveness.

In this section, promising imaging methods for characterization and monitoring of the TME will be reviewed. Current imaging methods that are available for potential use in the clinic include MRI, PET, SPECT, CT, and ultrasound. In addition, other optical imaging methods including photoacoustic imaging (PAI), intravital microscopy (IVM), bioluminescence, fluorescence, fluorescence molecular tomography (FMT), and optical coherence tomography (OCT) will be discussed. The goal is to present a high-level overview of current imaging methods used to characterize the TME relative to nanoparticle drug delivery.

\section{Magnetic resonance imaging}

\section{Introduction}

MRI allows for a nondestructive quantitative investigation of various aspects of the TME with good tissue contrast, spatial information, and sensitivity. Detailed, whole body anatomical images are obtained by employing a strong magnetic field and radio frequency (RF) electromagnetic waves. The protons in the human body normally have a scattered orientation; however, when they enter the magnetic field, the protons align with this field. The applied RF pulse causes the protons to become deflected by $90^{\circ}$ and store the energy of the RF pulse. After the RF pulse is suspended, the protons relax to their normal state and emit their stored energy where the time it takes for this phenomenon to occur differs depending on the type of tissue. Contrast agents can also be used to increase resolution and target specific cell types. The disadvantages of MRI as a technique for evaluating the TME include high costs and long acquisition times. MRI has the ability to reveal tumor treatment response of various sizes of nanoparticles by evaluating the EPR effect of a tumor. Karageorgis et al ${ }^{[41]}$ measured the functional parameters of eight different orthotopic tumor models which were correlated with optical imaging results of accumulation of three sizes of fluorescent nanoparticles. They found that comparing permeability and blood volume fraction with fluorescent accumulation of the nanoparticles allowed for differentiation of "EPR positive" and "EPR negative" tumors ${ }^{[4]}$. Magnetic nanoparticles can also be used to model colocalization of therapeutic nanoparticles, yielding valuable insight into the kinetics of nanoparticle distribution in tumor-bearing mice $^{[42]}$. Ramanathan et al. ${ }^{[33]}$ also demonstrated that the deposition characteristics of ferumoxytol iron nanoparticles in tumors may predict tumor response to nanoliposomal irinotecan in patients with solid tumors. Patients received ferumoxytol iron nanoparticles and the deposition characteristics and iron levels were quantified to demonstrate the EPR effect within tumor lesions. Treatment with nanoliposomal irinotecan followed and the tumor levels were measured at two separate biopsy locations. A positive trend was observed between ferumoxytol iron lesion values and irinotecan levels as well as tumor response ${ }^{[33]}$. These studies demonstrate the ability of MRI measurements of the distribution of iron nanoparticles to predict response to nanotherapy. 


\section{Extracellular matrix proteins and immune cells}

The development of advanced MRI techniques such as dynamic contrast-enhanced-MRI (DCE-MRI), diffusion-weighted MRI (DW-MRI), blood oxygen level dependent (BOLD) MRI, tissue oxygen level dependent (TOLD) MRI, and MRI-chemical exchange saturation transfer (CEST) have further increased the utility of MRI as an imaging modality for the TME. The use of DW-MRI, and various contrast agents in DCE-MRI, have allowed for the specific imaging of extracellular matrix (ECM) proteins including collagen $^{[43,44]}$ and hyaluronan ${ }^{[45,46]}$. These ECM proteins are recruited to the TME through activated fibroblast cells which are known to migrate toward the tumor. Tracking of fibroblasts has been achieved with MRI by pre-labeling fibroblasts ex vivo and then using magnetic resonance and optical probes to image the labeled fibroblasts post injection. The results showed recruitment of fibroblasts to the tumor and migration of the fibroblasts throughout the tumor ${ }^{[47,48]}$. Mesenchymal stem cells (MSCs), a fibroblast-like cell found surrounding blood vessels, have also been shown to migrate towards tumors. These cells have potential for repair of diseased or damaged tissue and are inherently tumor-homing and immunosuppressive, displaying many pro-tumor roles after arrival ${ }^{[49]}$. Tracking of MSCs labeled with superparamagnetic iron oxide nanoparticles in both malignant gliomas ${ }^{[40]}$ and pulmonary metastases ${ }^{[50]}$, and determining their use as vehicles for anti-tumor therapy ${ }^{[51]}$ have been performed using MRI, showing their tumor-homing response. Further use of iron nanoparticles as MRI contrast agents has resulted in imaging of tumor immune cells, specifically tumor-associated macrophages (TAMs). TAMs are also recruited to the TME, promote tumor progression ${ }^{[52]}$, and have been proven to be involved in phagocytosis and clearance of nanoparticle chemotherapeutics, and the tumor delivery of nanoparticles ${ }^{[53,54]}$. In addition, increased TAM presence in several types of human cancer have been associated with increased vascular density and worse clinical outcome. Thus, selective TAM imaging could guide treatment decisions and serve as a biomarker for long-term prognosis. Ultrasmall superparamagnetic iron-oxide nanoparticles (USPIO) are virus sized nanoparticles with an iron oxide core and a hydrophilic carboxydextran coating which, along with their size, allow them a much longer circulation half-life and help determine their pharmacology ${ }^{[55]}$. Iron exposure helps to regulate expression of iron transport related proteins, which are correlated to macrophage polarization states, suggesting that iron oxide nanoparticles would be preferentially phagocytosed by the multitude of TAMs in the TME ${ }^{[56]}$. In a study by Daldrup-Link et al ${ }^{[55]}$ results showed that FDA approved USPIO are preferentially phagocytosed by TAMs versus neoplastic tumor cells; however, uptake of the USPIO nanoparticles by the neoplastic tumor cells could be a confounding variable when preferentially targeting macrophages. Preferential accumulation of nanoparticles in TAMs as measured by MRI has also been used to study metastatic lymph nodes in prostate cancer ${ }^{[77-59]}$.

\section{Tumor vasculature}

MMPs are known to be involved in tumor angiogenesis and their activity has been evaluated in vivo using novel proteinase-modulated MRI contrast agents ${ }^{[60-63]}$. This novel proteinase-modulated MRI contrast technology was used in the development of theranostic nanoparticles (TNPs) which enabled enzymespecific drug activation at tumor sites and simultaneous in vivo MRI of drug delivery. The particles used ferumoxytol conjugated to an MMP-activatable peptide conjugate of azademthylcolchicine. The TNPs resulted in significant antitumor effects and tumor necrosis, demonstrating the potential of a nanotemplate that integrates tumor specificity, drug delivery, and in vivo imaging in a single moiety ${ }^{[63]}$. Other studies of MMP activity have utilized signal-amplifiable self-assembling (19) F MRI probes ${ }^{[64]}$ and an activatable cell-penetrating peptide covalently linked to cyclic-RGD, an integrin $a_{v} b_{3}$ binding domain which takes advantage of the interaction of MMP-2 with integrin $a_{v} b_{3}^{[65]}$. These $a_{v} b_{3}$, along with $a_{v} b_{5}$, integrins have more recently been imaged with superparamagnetic iron oxide loaded (SPIO) cRGD PEGylated polyion complex vessels in order to image the neovasculature in glioblastoma ${ }^{[66]}$. Modified SPIO in magnetoliposomes have also been used for targeting $a_{v} b_{3}$ integrins to study tumor angiogenesis ${ }^{[67]}$. Specific investigation of tumor vasculature can be achieved with various advanced MRI methodologies. The first is DCE-MRI which uses direct markers of angiogenesis such as $a_{v} b_{3}$ integrins ${ }^{[68]}$. The second methodology is dynamic susceptibility 
contrast (DSC)-MRI, which is the most commonly used MRI method for cerebral perfusion ${ }^{[69-71]}$. For both DSC-MRI and DCE-MRI, the contrast agent extravasates from the vasculature of leaky blood vessels in the tumor and the vessel size and density, vascular permeability and perfusion, and extravascular space can be assessed $^{[72-75]}$. DW-MRI is also used for evaluation of tissue perfusion; however, very complex quantitative analysis is required for this approach ${ }^{[71,76]}$. In order to acquire tissue blood flow and volume, arterial spin labeling BOLD-MRI is used. In BOLD-MRI methodologies, the visibility of the contrast is based on the concentration of deoxyhemoglobin, which is paramagnetic, and works best in poorly oxygenated tumors ${ }^{[77]}$. BOLD-MRI has demonstrated changes in tumor oxygenation following induction of angiogenesis ${ }^{[78]}$ and maps of functional vasculature ${ }^{[79]}$.

\section{Tumor hypoxia}

BOLD-MRI has also been used to image tumor hypoxia as a result of the leaky and tortuous vasculature. There are many clinical trials involving the use of agents to combat hypoxia and permit increased response to radiotherapy; however, tumor hypoxia for each patient should be measured in the clinic to determine eligibility for the studies. O'Connor et al. ${ }^{[80]}$ have used the fraction of tumor tissue refractory to oxygen challenge as a biomarker of hypoxia and demonstrated the ease at which this can be implemented in the clinic. A review of the comparison of BOLD-MRI and TOLD-MRI was completed by O'Connor et al. ${ }^{[81]}$ and described the theory and potential use of both methodologies to identify, spatially map, and quantify tumor hypoxia. Biomarkers derived from both BOLD-MRI and TOLD-MRI such as the dioxygen molecule $\left(\mathrm{O}_{2}\right)$ and the deoxyhemoglobin monomer $(\mathrm{Hb})$, can reveal underlying low $\mathrm{pO}_{2}$ and tissue hypoxia ${ }^{[81]}$. Several others have also demonstrated BOLD- and TOLD-MRI as prognostic biomarkers of response to therapy ${ }^{[82-84]}$. DCE-MRI has also proven useful for imaging tumor hypoxia where this methodology is routinely used for identification of tumors in patients, demonstrating the ease at which this could be implemented in the clinic ${ }^{[85-87]}$. Stoyanova et al. ${ }^{[85]}$ used an unsupervised pattern recognition technique which used a differential signal versus time curve associated with different TME characteristics in DCEMRI data to differentiate between well perfused, hypoxic, and necrotic regions of prostate cancer. With DCE-MRI already routinely used for tumor identification, this technique could be easily translated to the clinic $^{[85]}$ [Figure 1].

\section{Glycolysis and tumor $\mathrm{pH}$}

Under the hypoxic conditions in the TME, glycolysis is the predominant pathway for energy production leading to decreased $\mathrm{pH}$ of the extracellular space and inducing resistance of chemotherapies that are weak bases ${ }^{[88]}$. This glycolysis has been profiled with MRI in pancreatic ductal adenocarcinoma by Yamamoto et al. ${ }^{[89]}$ and in lymphoma by Rodrigues et al. ${ }^{[90]}$ who used ${ }^{13} \mathrm{C}$-MRI. The resulting anaerobic conditions lead to decreased $\mathrm{pH}$ in the TME in which many MRI methods have been developed for imaging this gradient in $\mathrm{pH}$. Gallagher et al ${ }^{[91]}$ and Scholz et al ${ }^{[92]}$ measured and quantified $\mathrm{pH}$ using ${ }^{13} \mathrm{C}$ labeled bicarbonate. More commonly used for tumor $\mathrm{pH}$ imaging is CEST-MRI which selectively saturates exchangeable protons that are transferred to bulk water signal ${ }^{[93,94]}$. Chen et al. ${ }^{[95]}$ has demonstrated accurate tumor $\mathrm{pH}$ measurements with parametric maps of CEST-MRI data and Longo et al. ${ }^{[96]}$ demonstrated a CEST-MRI approach to measuring $\mathrm{pH}$ using an X-ray contrast agent. To remove the influence of concentration on $\mathrm{pH}$ measurements, paramagnetic CEST (PARACEST)-MRI can be used ${ }^{[97-100]}$. It is also possible to differentiate between intracellular $\mathrm{pH}$ and extracellular $\mathrm{pH}$ with CEST-MRI. Intracellular $\mathrm{pH}$ detection methods utilize amine and amide concentrations from endogenous tissue proteins, which predominately remain in the intracellular space ${ }^{[101-106]}$. Exogenous molecules have also been utilized as extracellular $\mathrm{pH}$ reporters, permitting monitoring the effectiveness of novel anticancer treatments which reverse the glycolytic tumor phenotype ${ }^{[107-111]}$. The ability to translate CEST-MRI to the clinic still requires significant optimization; however, preliminary results and continued optimization studies for reducing respiration artifacts and expand the coverage of the body during an acquisition show promising results ${ }^{[96,112-117]}$. 

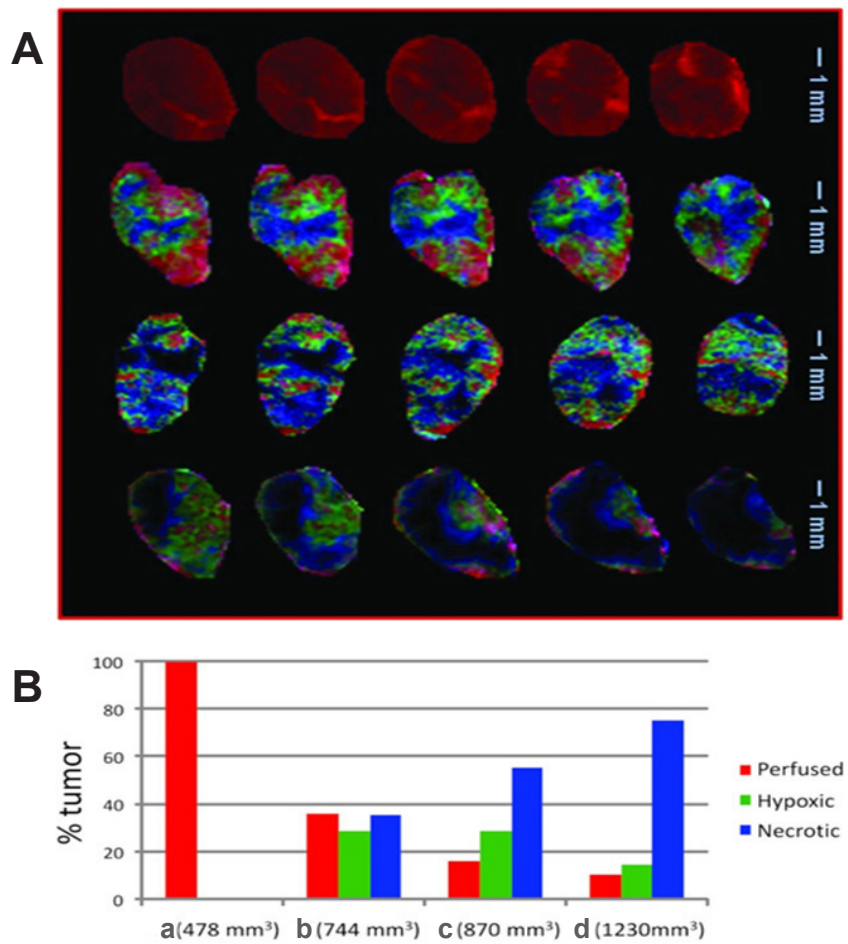

Figure 1. Study of well perfused, hypoxic, and necrotic regions of a prostate cancer tumor with dynamic contrast-enhanced mri with pattern recognition. (A) Composite color images of individual tumor subtissue features as identified by PR analysis. Images are presented for five tumor slices in four tumors with sizes: (a) $478 \mathrm{~mm}^{3}$, (b) $744 \mathrm{~mm}^{3}$, (c) $870 \mathrm{~mm}^{3}$, and (d) $1230 \mathrm{~mm}^{3}$. The perfused (red), hypoxic (green), and necrotic (blue/black) features are represented. (B) Tissue feature fractions in the four tumors. Figure reproduced with permission from ${ }^{[85]}$.

\section{Nuclear medicine}

\section{Introduction}

The two most commonly used imaging modalities in nuclear medicine are SPECT ${ }^{[118]}$ and $\mathrm{PET}^{[119]}$. Both modalities allow visualization of metabolic processes by detecting gamma rays through use of radiopharmaceuticals. The radiopharmaceutical is conjugated to a biologically active molecule (typically a sugar used for cellular energy) and injected into the vasculature. The detectors capture emissions from the radiopharmaceuticals and use software to create tomographic images of the concentrations in the body. These radiopharmaceuticals in SPECT imaging emit gamma rays while the radiopharmaceutical in PET imaging emit positrons. SPECT imaging offers a significantly cheaper option in terms of equipment and radio tracer costs as well as the possibility of greatly increased imaging time due to SPECT tracers typically having a much longer half-life. In contrast, PET offers the advantage of higher spatial resolution images, and the ability to quantify metabolic activity for accurate assessment of therapeutic effect. Although PET offers more radio tracers available specific to molecules in the TME, their production also requires availability of a cyclotron ${ }^{[118,119]}$. PET also offers excellent sensitivity to small concentrations providing biochemical information of diseases including cancer through molecular imaging ${ }^{[120]}$. Detection of isotopes with PET can be obtained down to the 100 picomolar level in target tissues, where this low concentration has little or no physiological effect, allowing the independent study of the target mechanism ${ }^{[121]}$.

\section{SPECT imaging}

PET is more commonly used for cancer imaging and diagnosis; however, SPECT has been previously used to image MMPs ${ }^{[62,122]}$, MSCs ${ }^{[51,123]}$, hypoxia ${ }^{[124]}$, and immune cells ${ }^{[125]}$. SPECT has also been used to monitor cancer nanotherapies such as imaging of prostate specific membrane antigen targeted nanoparticles that 
were radiolabeled with indium-111 along with their untargeted nanoparticle. Results showed that the targeted nanotherapy revealed an accumulation of $\sim 6 \% \mathrm{ID} / \mathrm{g}$ that remained constant over time whereas the untargeted nanoparticle had a higher tumor uptake of $\sim 8 \% \mathrm{ID} / \mathrm{g}$ but was cleared more quickly between 48 $96 \mathrm{~h}^{[35]}$. Other biodistribution studies of cancer nanotherapies demonstrate the increased circulation time of IV administered PEGylated liposomes ${ }^{[126]}$ along with the selective accumulation of technetium-99m $\left({ }^{99 \mathrm{~m}} \mathrm{Tc}\right)$ radiolabeled Caelyx ${ }^{\oplus}$ in the tumor compared to surrounding tissue ${ }^{[127,128]}$.

\section{PET imaging}

Similar to SPECT, PET offers the ability to image track the biodistribution of cancer nanotherapy including the analysis of ${ }^{64} \mathrm{Cu}$-labeledHER2-targeted PEGylated liposomal doxorubicin in patients with HER2 positive metastatic breast cancer showing their long circulation time and decreased accumulation in healthy tissue. The tracking of this nanotherapy also showed large heterogeneity of tumor accumulation in primary breast carcinomas and in different metastatic lesions ${ }^{[36]}$. This tracking of nanotherapy is an important application of PET and further demonstrates the application of imaging and characterization of the TME in determining factors dictating the response to nanoparticle treatment. In other experiments, the application of PET zirconium-89 nanoreporter to predict response to nanotherapy was investigated. This study showed correlation of the tumor uptake of zirconium-89 nanoreporter with the tumor uptake of various other nanoparticle drugs. This demonstrated that the zirconium-89 nanoreporter could be used as a surrogate measure of nanoparticle tumor delivery and used as inclusion criterion for patient's tumor that is amenable to nanotherapy ${ }^{[129]}$. PET has also been used for imaging MMPs ${ }^{[130-134]}, \operatorname{MSCs}^{[135-138]}$, and immune cells ${ }^{[139-141]}$. Choline-phospholipid metabolism PET imaging has been demonstrated by several groups and successfully translated to the clinic, despite limitations in metabolite discrimination ${ }^{[142-145]}$. PET imaging is also very useful for measuring tumor vasculature and lymphatics through quantitative measurement of blood flow and perfusion ${ }^{[146-148]}$, blood volume and vascular permeability ${ }^{[149,150]}$, and specific molecular markers of tumor vasculature including integrins ${ }^{[151-156]}$, VEGF receptors, and EGF receptors ${ }^{[157-165]}$.

\section{Tumor hypoxia and $p H$}

PET offers much more advanced imaging of tumor hypoxia ${ }^{[166,167]}$. The most commonly used PET tracer for hypoxia imaging is FMISO $^{[168]}$ which has been used in patients with glioma ${ }^{[169,170]}$ and in mice with micrometastases $^{[135]}$. When compared with other PET tracers for imaging tumor hypoxia, studies showed that each tracer had advantages that could be used depending on the desired imaging requirements ${ }^{[171,172]}$. PET imaging also has the capability of detecting changes in hypoxia before and after treatment, providing insight on treatment response ${ }^{[173-175]}$. Various materials have been investigated for use in PET hypoxia imaging including copper ${ }^{[176]}$ and gallium ${ }^{[177]}$, where copper demonstrated faster accumulation while gallium demonstrated minimal liver accumulation and excellent specificity to hypoxic tissue. Changes in hypoxia and glycolysis in the TME result in changes in $\mathrm{pH}$ where a few PET probes for $\mathrm{pH}$ have been developed; however, research in this area is limited due to the limited spatial resolution ${ }^{[178-181]}$. Under hypoxic conditions, glycolysis is the major energy producing pathway making glycolysis an attractive target for therapeutic interventions. Moreover, glycolytic inhibitors that cause ATP depletion show promising anticancer activity and regressions in in vivo models of solid tumors ${ }^{[182]}$. Monitoring glycolysis using PET has been demonstrated; however, only the early stages of glycolysis are probed, where more characterization is needed to evaluate complete metabolism ${ }^{[89,183-185]}$. Nuclear medicine has demonstrated broad use in imaging multiple factors within the TME, further demonstrating the abilities for correlating factors within the TME to successful delivery of nanotherapies ${ }^{[129]}$.

\section{Computed tomography}

\section{Introduction}

$\mathrm{CT}$ is a noninvasive tomographic imaging technique based on $\mathrm{X}$-ray attenuation that permits high efficiency and fast temporal resolution related to the electron density of tissue. The X-ray images are 
generated with a fan-shaped X-ray beam and digital X-ray detector that rotate about the patient to collect multiple X-ray projections needed to generate a tomographic CT image. The image processor then performs massive calculations to construct the CT image. A limitation of CT in imaging soft tissue lesions such as tumors, is a lack of contrast between the soft tissue and normal tissue, therefore contrast agents are necessary to achieve accurate tumor detection ${ }^{[186]}$. Clinically, iodinated small compounds are used as a CT contrast agent, but are usually limited by short circulation time, potential adverse effects, and lack of specificity ${ }^{[187]}$. However, Sahani et al. ${ }^{[188]}$ used CT contrast agents allowing the assessment of tumor vascularity and perfusion in rectal cancer where tumors showed higher blood flow and shorter mean transit time which reversed after treatment with chemotherapy and radiation therapy. Additionally, the study indicated that initial high blood flow and short mean transit time typically responded poorly to chemotherapy and radiation therapy ${ }^{[188]}$. To improve circulation time, specificity, and visualization of CT contrast agents in the tumor vasculature, nanoparticle contrast agents have been explored, allowing not only investigation of the tumor vasculature, but also how the nanoparticles interact with the TME ${ }^{[189-191]}$. Ghaghada et al. ${ }^{[191]}$ showed that the longer circulation time of nanoparticle CT contrast enabled longitudinal tracking, revealing regions with high 'leakiness' and increased vessel permeability within tumors. The extravascular signal enhancement of these nanoparticle CT contrast agents showed highly heterogeneous signals within all tumors and also varied between animals ${ }^{[191]}$. In recent years, there has been even more advancement in CT contrast agents with the development of negative CT contrast agents as well as spectral CT, making it feasible to use functional CT contrast nanoagents (FCTNAs) for evaluation of the $\mathrm{TME}^{[192-194]}$. These FCTNAs can be targeted to specifically bind to overexpressed receptors in the TME for imaging purposes and for targeted drug delivery. Zou et al ${ }^{[195]}$ used targeted CT contrast to accumulate at an $\mathrm{a}_{\mathrm{v}} \mathrm{b}_{3}$-integrin positive B16 melanoma, which once degraded, released the loaded doxorubicin resulting in suppression of the B16 melanoma. Targeted CT imaging has also been achieved for different cancer types, including human hepatocellular carcinoma through binding to the overexpression of asialoglycoprotein receptors $^{[31]}, 4 \mathrm{~T} 1$ breast tumors through overexpressed p32 cell surface receptors ${ }^{[196]}$, and for the over- or under-expression of $\mathrm{a}_{\mathrm{v}} \mathrm{b}_{3}$ integrin in various tumor types ${ }^{[197]}$.

\section{Tumor hypoxia and $p H$}

Another use of FCTNAs involves contrast that is designed to diagnose, determine different levels of, and treat tumor hypoxia, offering improved prognosis and decreased treatment resistance ${ }^{[198-201]}$. When a tumor is hypoxic, this often leads to changes in $\mathrm{pH}$ where FCTNAs have been developed to respond to the acidic microenvironment of the tumor. Tian et al. ${ }^{[30]}$ developed a FCTNA that, through interaction with the acidic TME, folds and inserts into the lipid bilayer of the tumor cells. Negative CT contrast agents have also been designed to generate hydrogen in response to the acidic TME for accurate diagnosis of osteosarcoma ${ }^{[193]}$ [Figure 2]. In order to enhance the effectiveness of $\mathrm{pH}$ responsive contrast agents, FCTNAs have also been conjugated to respond to glutathione ${ }^{[31,202,203]}$. Although conventional CT may be limited, it has great advantages of high spatial and temporal resolution and new nano-CT contrast agents have improved the CT technology allowing for improved monitoring of the TME, although it is important to note that relatively high local concentrations of contrast are needed to achieve good sensitivity ${ }^{[204]}$.

\section{Ultrasound}

Introduction

Ultrasound is a widely used diagnostic tool for visualizing the structure of native tissues which uses backscattered radio-frequency sound waves to generate a gray-scale image, resulting in a qualitative image of tissue structure. The ultrasound beam is created through mechanical oscillations of crystals in a transducer that is excited by electrical pulses and converts the energy into sound. The sound waves are detected as reflected echoes after propagation through tissue where the reflections occur at the boundary between tissues with varying densities. This leads to the altered physical tissue characteristics in disease states being readily visualized. Conventional and advanced ultrasound techniques are excellent probes for 

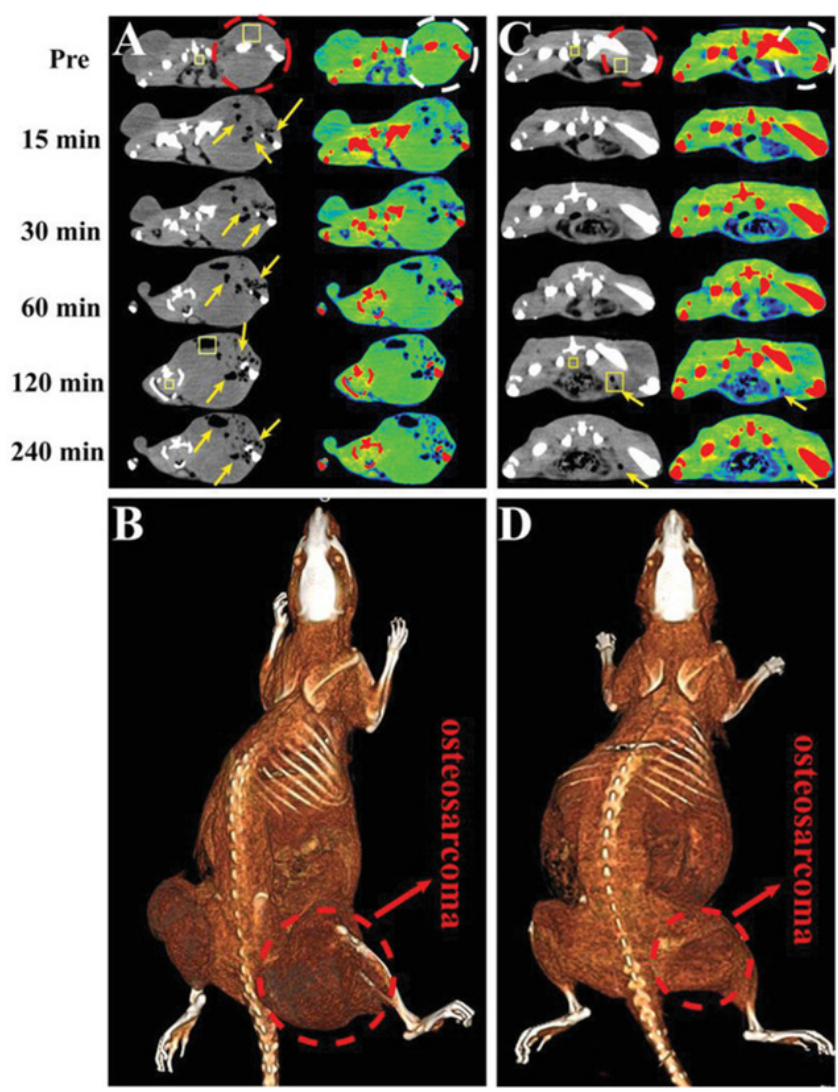

Figure 2. Computed tomography (CT) images using a negative $\mathrm{CT}$ contrast agent designed to generate hydrogen in response to acidic $\mathrm{pH}$ in the tumor microenvironment of osteosarcoma. (A, C) CT images of osteosarcoma-bearing rats by intratumor and intravenous injection of HMSN@AB@PEG nanoparticles (at various time points). Transverse gray and corresponding pseudo color images were obtained with a dashed line representing the area of osteosarcoma and the region of arrows was the low CT density area of $\mathrm{H}_{2}$ release. The rectangle ROI were used to calculate the variation of $\mathrm{CT}$ density between two time points (Pre and $120 \mathrm{~min}$ ). (B, D) Corresponding intratumor and intravenous 3D reconstruction maps of rat with a dashed line representing the area of osteosarcoma. Figure reproduced with permission from ${ }^{[193]}$.

monitoring and correlating factors within the TME due to cost effectiveness, ease of use, lack of ionizing radiation, non-invasiveness, portability/accessibility of the equipment, and the ability to multiplex. Recent work has focused on the use of nanotechnology in combination with ultrasound to increase the localized delivery of drugs to solid tumors ${ }^{[205]}$. The leaky and tortuous vasculature within the TME prevents accumulation of the nano-drug; however, an ultrasound process called sonoporation can increase the size of pores in the cell membrane after mechanical impact of ultrasound radiation, increasing permeability and allowing passive entry of nanoparticles into cells ${ }^{[205]}$. Ultrasound can also be used to release drug from nanoparticles. For example, ultrasound induced shear stress can be used to rupture nanoparticles (e.g., drug loaded polymersome, perfluorocarbon nanoemulsions, and liposomal nanocarriers), releasing their cargo at the targeted site ${ }^{[206]}$. Thus, sonoporation of the endothelial cells and shear stress of the nanoparticle drugs after interaction of ultrasound radiation allows for increased cell permeability, cell retention of the drug, stimulation of drug release only at the targeted site, and decreased off-target toxicity ${ }^{[205,207]}$. The field would benefit from increased depth imaging and improved quantification to allow increased translation of these advanced techniques into the clinic. Over the past several decades, many researchers have developed techniques to improve the ability to make tissue characterization with ultrasound quantitative ${ }^{[208-211]}$. Lizzi et al. ${ }^{[212]}$ and Ghoshal et al ${ }^{[208]}$ performed analytical investigations of specific tissue features that determine spectral signatures. These advancements in ultrasound imaging have resulted in successful quantification of collagen, a major component of the TME and barrier to drug delivery to tumors ${ }^{[213-216]}$. 

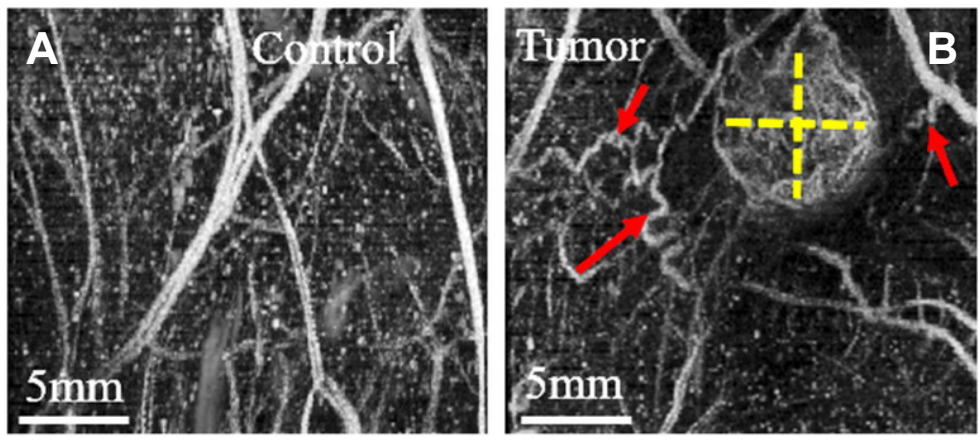

Figure 3. Acoustic angiography images of angiogenesis and vessel tortuosity. (A) Images of control non-tumor bearing regions and (B) fibrosarcoma tumor bearing regions in a rat. Tumor size is represented with dashed yellow lines. Tortuous vessels denoted by red arrows represent angiogenesis around the periphery of the tumor. Higher vascular density in the tumor is demonstrated by more enhancement in the images due to higher contrast agent perfusion. The vasculature in control images is more linear and directional, as shown. Figure reproduced with permission from ${ }^{[217]}$.

\section{Tumor vasculature}

Other advancements in ultrasound imaging including the development of acoustic angiography based on the use of ultrasound contrast agents, called microbubbles, have resulted in visualization of tumor microvascular architecture without a significant contribution from background tissues ${ }^{[218]}$. Microbubbles are spherical cavities with a gas encapsulated in a shell made of phospholipids, surfactant, denatured human serum albumin, or synthetic polymer which resonate with ultrasound waves. Microbubbles provide a strongly reflective interface, and are currently clinically approved for use as an ultrasound contrast agent in cardiac and liver imaging ${ }^{[219]}$. These microbubble contrast agents are confined to the blood stream when injected intravenously. Acoustic angiography has been used to map micro-vascular networks in the $\mathrm{TME}^{[218,220-222]}$, differentiate tumor tissue from healthy tissue ${ }^{[217,221]}$ and various tumor types ${ }^{[223]}$, as well as monitor response to therapy ${ }^{[37,224]}$. The dense and tortuous vessel network in solid tumors allow the use of acoustic angiography in the assessment of neo-vascularization for early diagnosis ${ }^{[217]}$ [Figure 3]. One limitation of acoustic angiography for vascular imaging is the imaging depth (up to several centimeters ${ }^{[218]}$ ). However, this can be overcome by using techniques such as Doppler-based ultrasound techniques ${ }^{[225]}$ and ultrasound localization microscopy ${ }^{[226]}$, although these methods are susceptible to tissue motion. In order to increase specificity of ultrasound imaging to target molecules, microbubbles conjugated to antibodies or ligands are used where the molecular ultrasound targets are expressed on vascular endothelial cells. These microbubbles have greatly reduced adverse effects when compared to CT/MRI contrast agents with an added advantage of real-time imaging ${ }^{[37]}$.

MMPs are involved in angiogenesis, tumor invasion, and metastasis where ultrasound microbubbles have been conjugated with specific antibodies for MMP imaging ${ }^{[227]}$. Other angiogenic markers are vascular endothelial growth factor receptor 2 (VEGF-2) and $\mathrm{a}_{\mathrm{v}} \mathrm{b}_{3}$ integrin receptor, which are both hallmarks of the neovasculature that occurs in tumors at an early stage to establish an independent oxygen and nutrient supply. Targeted microbubbles for VEGF-2 have been developed where imaging studies show expression of the receptor in several different tumor models ${ }^{[228-231]}$. Along with molecular targeting of vascular markers, microvasculature imaging is also an excellent tool in assessment of tumor malignancy and response to treatment such as predicting response to therapy before measurable changes in tumor volume ${ }^{[37]}$. Vessel tortuosity, or vessels that contain abnormal twists and turns leads to abnormal or variable drug distribution of nanoparticle drugs within the tumor. Lindsey et al ${ }^{[232]}$ have assessed the combined microvascular and molecular imaging in a technique termed molecular acoustic angiography, suggesting that increased distance from VEGFR2- and selectin-targeting sites showed decreased vessel tortuosity. Lakshman et al. ${ }^{[233]}$ performed screening studies of the tumor vasculature in the TME of an orthotopic mouse model of human 
pancreatic cancer and demonstrated heterogeneous microvascular distribution with high perfusion at the periphery of the tumor and a poorly perfused core.

\section{Ultrasound elastography}

Ultrasound elastography is an advanced ultrasound method available for assessing factors within the TME. Elastography has the ability to measure tissue pressure and stiffness by visualizing the shear modulus of tissue, an intrinsic property of soft tissue defined as the ratio of shear stress to shear strain. For measurement of shear modulus, tissue motion is induced by using quasi-state, harmonic, or transient mechanical source. The mechanical movement or spatial variation of the tissue response is measured with an ultrasound transducer in which a simplified or continuum mechanical model is applied to obtain shear modulus values ${ }^{[234]}$. The pressure gradient at the tumor margin exerts mechanical stress which creates an increase in shear modulus ${ }^{[235]}$. Relative to the study of cancer, initially, ultrasound elastography has been primarily used for differentiating benign tumors from malignant ${ }^{[236-239]}$; however, more recently it has been used for the study of various factors within the TME. The correlation of cancer associated fibroblasts in breast cancer with markers of aSMA and CD34, has been investigated using ultrasound elastography finding that aSMA was positively associated with elastography scores and elevated in malignant lesions, while CD34 was negatively associated with elastography scores and is downregulated in malignancies ${ }^{[240]}$. Several other studies have shown increased collagen fibers with increased shear modulus ${ }^{[241-243]}$. In regards to drug delivery, elastography has been used to map the relationship between shear modulus and drug delivery within the pancreatic ductal adenocarcinoma TME. Results showed that drug delivery was directly influenced by shear modulus and that the factors within the TME including collagen modulate the shear modulus values ${ }^{[24]}$.

\section{Optical imaging techniques}

\section{Introduction}

Current clinically available imaging techniques including PET, MRI, and CT typically have low spatial resolution, therefore, it is difficult to use these techniques for any real-time in vivo analysis of genetic, molecular, and cellular events ${ }^{[245-24]}$. Optical imaging methods are those that use luminescent enzymes such as bioluminescence or fluorescent proteins and dyes where these have been largely used for in vitro and ex vivo applications in cellular and molecular biology. These methods have the advantage of speed and versatility as well as the lack of special equipment, allowing the technique to be performed in any research lab or clinic ${ }^{[248]}$. Although these techniques are limited by depth of penetration (i.e., millimeter range), the ability to image the functional dynamics of the TME relative to time and space would give information in predicting and monitoring response to therapy.

\section{Photoacoustic imaging}

Photoacoustic imaging, or PAI, uses pulses of light to illuminate tissue, which causes a pressure change when absorbed, generating ultrasound waves that can be detected with an ultrasound transducer. To allow the spatial resolution, temporal resolution, imaging depth, and image contrast to be tuned in PAI, light sources varying in wavelength can be used. Wavelengths in the visible and near-infrared region primarily takes advantage of the contrast due to hemoglobin, although other contrast agents including dyes or genetically expressed absorbers are used to obtain targeted molecular contrast. PAI has been used to quantify nanodrug delivery by conjugating the particles with molecules that are oxidized during release to produce a concentration-dependent photoacoustic signal ${ }^{[249]}$. Additionally, PAI is used for visualizing the development of vasculature in tumors including tumor vessel tortuosity ${ }^{[250]}$ [Figure 4], vessel diameter and density ${ }^{[251]}$, and the recruitment of existing vessels to feed the tumor mass ${ }^{[250,252]}$. Tumor vasculature characteristics are imaged by using a single wavelength of light, selected as an isosbestic point of $\mathrm{Hb}$ and $\mathrm{HbO}_{2}$ at $532 \mathrm{~nm}$ and permits sub-100-mm resolution, non-invasively. Total hemoglobin concentration blood oxygen saturation $\left(\mathrm{sO}_{2}\right)$ can be measured with PAI in combination with spectral 

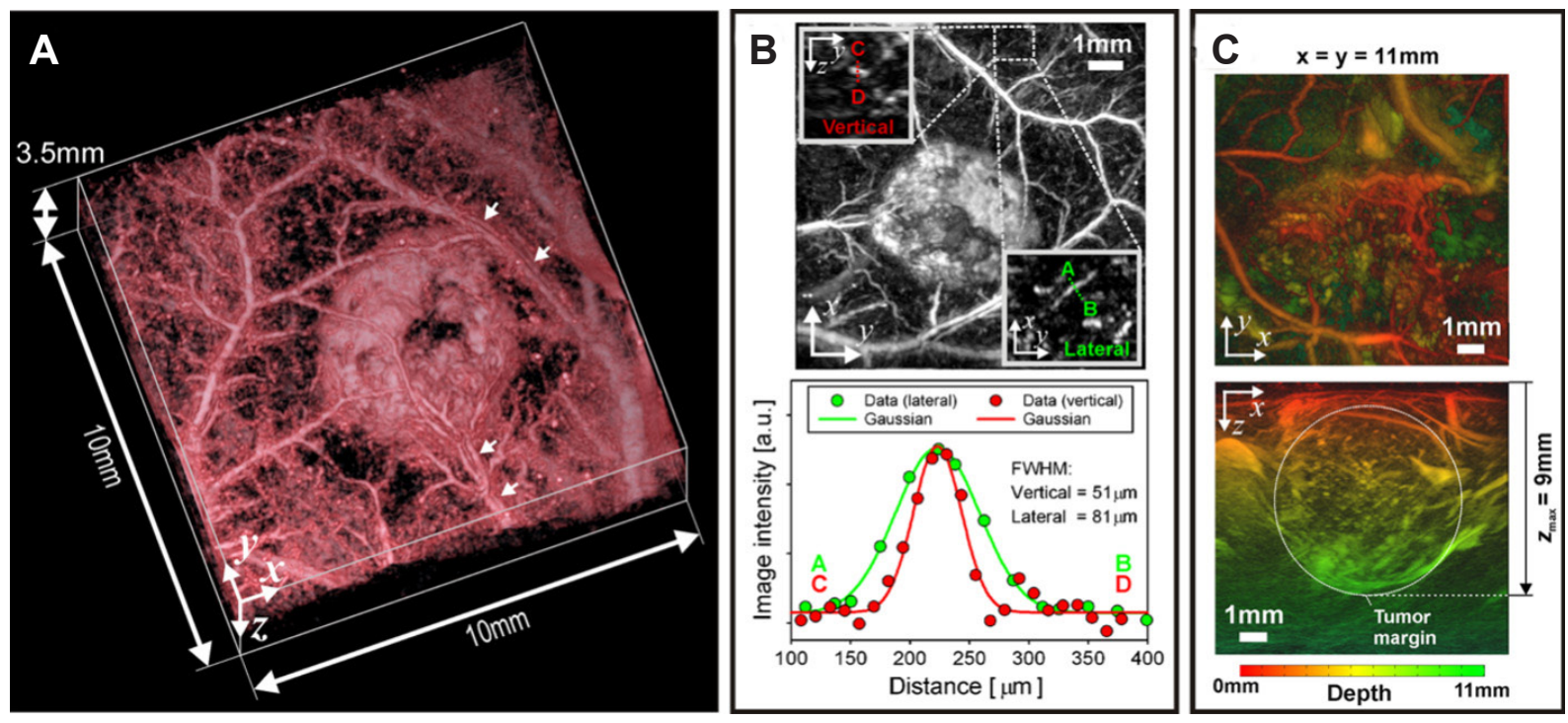

Figure 4. Tumor vasculature development and vessel tortuosity using photoacoustic imaging. (A) Volume-rendered 3-D photoacoustic image of a LS174T subcutaneous tumor (day 8 post inoculation) with arrows indicating artery-vein pairs. (B) Top: Maximum intensity projection (MIP) of the image presented in (A). The insets show magnified $y-z y-z$ and $x-y x-y$ MIPs of a region containing a small blood vessel. Bottom: Profiles across this vessel between points A-B and C-D were plotted and fitted with a Gaussian function to obtain spatial resolution. (C) Photoacoustic images of a larger SW1222 subcutaneous tumor (day 12 post-inoculation) illustrating an imaging depth of at least $9 \mathrm{~mm}$. Figure reproduced with permission from ${ }^{[250]}$.

unmixing algorithms where total hemoglobin concentration is typically higher and $\mathrm{sO}_{2}$ is typically lower in tumor tissue compared to normal tissue ${ }^{[253-257]}$. Low $\mathrm{sO}_{2}$ values are due to typical tumor hypoxia leading to poor perfusion and high consumption of $\mathrm{O}_{2}$ which can also be correlated with vascular maturity and perfusion efficiency ${ }^{[258-261]}$. In contrast, decreasing total hemoglobin concentration has been shown to indicate vessel normalization following anti-angiogenic therapies ${ }^{[262]}$ and increasing $\mathrm{sO}_{2}$ has been shown to predict radiotherapy response in mice ${ }^{[263,264]}$. Complementing the investigation of vascular features, tumor hypoxia ${ }^{[265]}$ and tumor $\mathrm{pH}^{[95,266,267]}$ can also be evaluated.

\section{Extracellular matrix proteins and immune cells}

Aside from tumor vasculature and oxygenation, many other components can be evaluated with PAI including collagen, MMPs, and immune cell infiltration. Preclinical studies of fibrosis and lipids has mainly focused on other diseases including Crohn's disease ${ }^{[268,269]}$ and atherosclerosis ${ }^{[270,271]}$, but show excellent promise for its application in the TME. The monitoring of MMP production or activity in the TME provides a potential predictive biomarker of metastasis and their production has been monitored preclinically with $\mathrm{PAI}^{[272-275]}$. By using exogenous contrast agents, immune cells can be labeled and tracked, in vivo, and this has been shown with fluorescently labelled $\mathrm{T}$ cells ${ }^{[276,277]}$. Other contrast agents including organic semiconducting polymer nanoparticles and small-molecule dyes have been used to label and track injected stem cells ${ }^{[39,278]}$ where this pre-loading of cells avoids the macrophage phagocytosis of the nanoparticles ${ }^{[279]}$. The organic semiconducting nanoparticles in the far infrared window revealed significant photoacoustic contrast enhancement, demonstrating feasibility for real-time photoacoustic monitoring of stem cell behaviors such as cell differentiation which would advance the understanding of stem cell-based therapy ${ }^{[39]}$.

\section{Intravital microscopy}

Intravital microscopy allows for in vivo imaging of cancer at subcellular-scale resolutions. This nonlinear microscopy relies on scanning tissue with single photon or multiphoton fluorescent microscopy and 


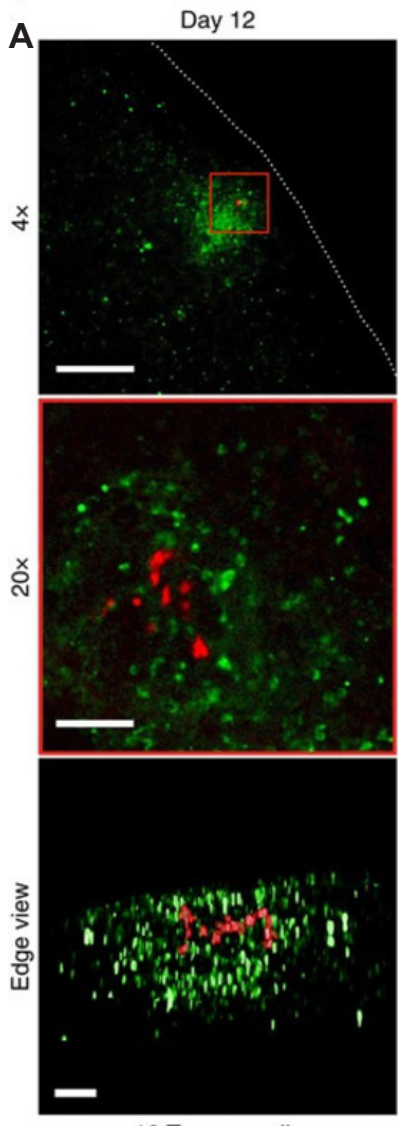

16 Tumour cells

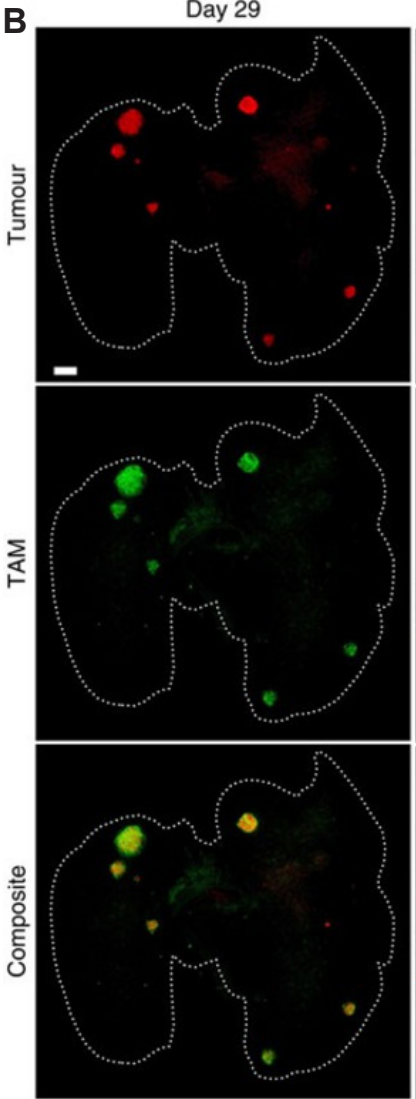

Tumour

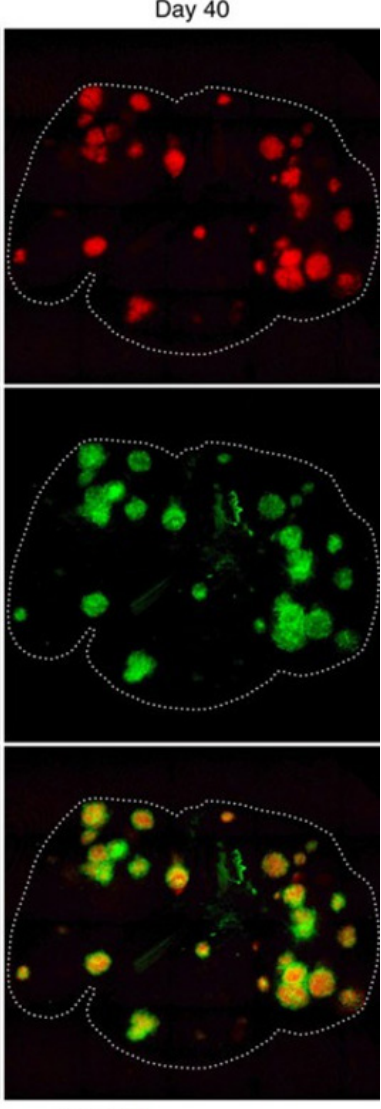

TAM 탐

Figure 5. Investigation of the large heterogeneity in tumor macrophages and tumor size with intravital microscopy. (A). Early-stage tumors with clear indication of surrounding tumor associated macrophage (TAM) presence. TAM infiltration is observed in response to 16 individual tumor cells within a single nascent nodule. Scale bars, $1000 \mathrm{~mm}$ (top), 100 mm (middle/bottom). (B) Mouse tumor analysis at 2 stages of disease progression revealing facile tumor detection at varying locations and heterogeneous distribution of TAM. Dashed lines outline the lung. Scale bars, $1000 \mathrm{~mm}$. For all, days denote time post inoculation. Figure reproduced with permission from $^{[34]}$.

is commonly used to image the extracellular matrix in cancers ${ }^{[280]}$. To form the image, the excitation beam interacts with the target molecule to excite a transition between two energy levels. The light that is emitted upon relaxation of the target molecule is due to vibrational and rotational motion and this light is detected to generate the final image ${ }^{[281]}$. Most studies have been completed preclinically, with clinical applications being limited to endoscopic investigation of gastrointestinal cancers ${ }^{[282]}$ and cytoscopic investigation of bladder tumors ${ }^{[283]}$. Preclinically, advanced fluorescent labeling techniques can be used with IVM to characterize the TME in relation to tumor vasculature growth, regression, and density ${ }^{[284,285]}$, as well as metastasis ${ }^{[286]}$, tumor-associated immunocytes ${ }^{[287]}$, the interaction of cancer therapeutic agents with cancer and immune cells ${ }^{[288]}$, and the response of macrophages to neoadjuvant chemotherapy ${ }^{[289]}$. Often, a window of dorsal skinfold chamber is installed on animals with superficial tumors including skin tumors for IVM imaging, where deeper tumors such as colon, liver, or pancreatic tumors are not directly accessible, requiring specific surgical procedures to expose the tumor ${ }^{[290,291]}$. The heterogeneity of immune components such as macrophages can lead to large variances in prognosis. Cuccarese et al. ${ }^{[34]}$ studied the heterogeneous distribution of macrophages between tumors showing large heterogeneity between tumor sizes which was later correlated to nanotherapeutic drug delivery [Figure 5]. The authors found that the uptake of nanotherapeutics into the tumor were dependent upon macrophage density, where macrophage depletion decreased nanotherapy delivery and efficacy ${ }^{[34]}$. Macrophages have also been shown to mediate 
the antitumor activity of bisphosphonates in $4 \mathrm{~T} 1$ mouse mammary tumors ${ }^{[292]}$. There have also been studies that employed nanotechnologies, referred to as "smart" nanotechnologies, that use fluorophores or quenchers which respond to changes in the TME conditions ${ }^{[293]}$. One limitation of IVM is overlap or bleed-through between related fluorophores and when using particular laser light sources and microscope filter sets while labeling and imaging more than one component of the TME. To overcome this limitation, fluorescence-lifetime imaging microscopy can be used in which fluorophores with different fluorescence lifetimes are used ${ }^{[294]}$ and this can be combined with standard wavelength filtering to allow investigation of TME dynamics ${ }^{[295,296]}$.

\section{Bioluminescence imaging}

Bioluminescence uses ultra-sensitive charge-coupled device cameras to measure biopermeable luminescence after administration of a luciferase gene such as ${ }_{\mathrm{D}}$-luciferase of coelenterazine. Luciferases proteins are found naturally in insects, bacteria, and plants and have enzymatic activity that catalyzes the oxidation of the substrate in a reaction in the presence of ATP, oxygen, and luciferin resulting in the emission of a photon. The resulting photons can travel through tissue and thin bones and are detected to form the image. This process allows the visualization of the movement of cancer cells in living animals ${ }^{[297-299]}$. Bioluminescent nanoprobes have been developed for the in vitro analysis of MMP activity composed of AuNPs and luciferases, where the AuNPs efficiently quenched the bioluminescent emission allowing the nanoprobe to detect the proteolytic activity of MMP- $2^{[300]}$. Other in vitro bioluminescent probes have been developed in combination with quantum dots allowing for multiplexing of different MMP activities and increased detection sensitivity ${ }^{[301,302]}$. In vivo bioluminescent detection of MMP activity has been achieved by using luciferase-quenched protein nanoprobes bound to collagen, allowing them to remain in the extracellular space for increased sensitivity ${ }^{[303]}$. Bioluminescent probes have also been used to track MSCs in vivo ${ }^{[304-307]}$, in vitro ${ }^{[308]}$, and ex $v i v o^{[309]}$, as well as immune cells ${ }^{[310]}$. Tumor hypoxia and altered $\mathrm{pH}$ were shown to reduce bioluminescent signals ${ }^{[311,312]}$ which led to the development of specific bioluminescent probes for tumor hypoxia using CYP450 reductase ${ }^{[313]}$ and a HIF-1a reporter construct ${ }^{[314]}$. Due to the advantage of bioluminescence for visualizing movement of cells, it is also an excellent tool for the analysis of tumor-stroma interactions and has been used with heterogeneous 3D models for drug screening ${ }^{[315,316]}$.

\section{Fluorescence imaging}

Fluorescence imaging excites the atoms in the target molecule to a higher energy level and when they relax to a lower level, they emit photons at a lower energy but longer wavelength than the excitation light. The emitted light is filtered from the excitation light based on wavelength before sent to the detector to form an image. Fluorescence imaging has an increased spatial and temporal resolution in comparison with bioluminescence imaging and has the ability to detect single cells in real time ${ }^{[24,317]}$. Fluorescent proteins can effectively transfect cancer cells, allowing visualization of the movement of cancer cells through vasculature, in vivo, demonstrating the ability to image processes such as metastasis and intravasation or extravasation of cancer cells ${ }^{[318]}$. By using multicolor fluorescent imaging, cancer cells can be distinguished from stromal cells in the $\mathrm{TME}^{[319,320]}$ and the interactions between the cancer cells and the tumor stroma can be elucidated ${ }^{[321,322]}$. Growth factors including the Ras superfamily G protein ${ }^{[323]}$ and VEGF which promotes angiogenesis ${ }^{[324]}$ have been fluorescently imaged, showing the promise of this method for analysis of cell signaling. Further studies of tumor angiogenesis using fluorescent imaging has been performed using a near infrared probe that labels blood vessels to evaluate changes over time ${ }^{[325]}$. Other fluorescent studies of tumor vasculature include combination with multispectral unmixing ${ }^{[326]}$, the use of Cy5.5-labeled probe for $\mathrm{CD} 13$ expression on tumor neovessels ${ }^{[327]}$, the use of a VEGF conjugated infrared dye ${ }^{[328]}$, and the use of dynamic fluorescent imaging for monitoring vascular density, perfusion rate, and permeability, simultaneously ${ }^{[329]}$. Similar to bioluminescence imaging, there are excellent fluorescent nanoprobes for imaging MMP activity that are "switched on" by the enzyme activity when they bind to the MMP target. 
The design of these probes involves the use of quantum dots conjugated to peptides ${ }^{[32,330,331]}$ or the use of silica nanoparticles ${ }^{[332]}$ and gold nanoparticles ${ }^{[331,333,334]}$. Fluorescent probes have also been designed to investigate the accumulation of hyaluronan and collagen in ex vivo tumor sections ${ }^{[335}$ and for in vivo intraoperative imaging of hyaluronic acid conjugates for assistance in surgical resection of pancreatic cancer ${ }^{[336]}$. Both MSCs and immune cells may be labeled ex vivo with fluorescent molecules to determine the localization and quantification of immune cells in whole tissue ${ }^{[337]}$ or to inject the prelabeled cells and view their activity in $v i v{ }^{[338,339]}$. Quante et al. ${ }^{[340]}$ showed evidence that CAFs originate from bone marrow and derive from MSCs through the expression of red fluorescent protein in aSMA-RFP transgenic mice. Often, investigations of CAFs are performed using immunofluorescence, in vitro ${ }^{[341,342]}$; however other studies have shown the expression of fibroblast activation protein on $\mathrm{CAFs}^{[343]}$. To study hypoxia in the TME, several fluorescent probes have been designed, primarily involving the use of near infrared probes conjugated to hypoxia activatable molecules, demonstrating a turn-on fluorescent probe ${ }^{[34-347]}$. Activatable fluorescent probes are also used to monitor $\mathrm{pH}$ in the tumor microenvironment ${ }^{[348-351]}$. Fang et al. ${ }^{[349]}$ designed a fluorescent probe that responds to the acidic nature of the tumor microenvironment by using a coumarin-hybridized dye with spirolactam ring structure which remains in a ring-closed form at neutral $\mathrm{pH}$, displaying fluorescent peaks in the visible region but the rings opens up at acidic $\mathrm{pH}$, displaying fluorescent peaks in the near infrared region. These probes are excellent for identifying the tumor. Liu et al. ${ }^{[350]}$ developed a fluorescent probe based on CdSe quantum dots where the reduced tumor $\mathrm{pH}$ leads to the loss of the surface stabilizer of the quantum dot, changing the fluorescence intensity and a mathematical model between fluorescence intensity and $\mathrm{pH}$ reveals the $\mathrm{pH}$ of the tumor environment within a range of 6.1-7.8. Optical imaging methods are advantageous in their increased spatial and temporal resolution as well as the ability to image multiple targets simultaneously; however, the depth of imaging is still limited as well as the topographical information.

\section{Fluorescence molecular tomography}

FMT takes advantage of the diffuse nature of photon propagation in tissue, producing a tomographic reconstruction in $3 \mathrm{D}$ through combining micro-CT and fluorescence images. Similar to fluorescence imaging, a fluorescent agent is injected and accumulated in targeted tissues. The fluorescent agent is excited with a laser, emitting photons with a longer wavelength than the excitation wavelength allowing them to be filtered and detected. With FMT, multiple spatial locations on the tissue are illuminated allowing a 3D reconstruction of the target tissue ${ }^{[352]}$. A main focus of FMT has been the imaging of MMPs including macrophages ${ }^{[353]}$. Activatable probes analyzed with FMT have been used to monitor the greatly upregulated MMP in glioblastoma by injecting a MMP-750 probe followed by acquiring a micro-CT and fluorescence images for the reconstruction of $\mathrm{FMT}^{[354]}$. The results allowed for accurate location detection of orthotopic glioma mouse tumors. MMPs have been shown to play a large role in angiogenesis, where FMT has been used to evaluate the volume of tumor vasculature in mice as well as the normalization of tumor vasculature over time after treatment with antiangiogenic agents ${ }^{[355,356]}$. A good correlation between the degree of tumor vascularization and the degree of tumor accumulation was found with FMT, suggesting that FMT can be used not only to characterize and predict enhanced accumulation of nanoparticle drugs but can be used to pre-select patients that are likely to respond to passively tumor-targeted nanomedicine treatments ${ }^{[357]}$. The advantages of FMT include its inherent quantitative nature, depth of penetration, ability to image without any ionizing radiation, and its ability to multiplex by using fluorophores that do not overlap all demonstrating the potential abilities of FMT for evaluation of the tumor microenvironment ${ }^{[358]}$.

\section{Optical coherence tomography}

OCT is a relatively new, non-invasive imaging technique that provides label-free imaging of living tissue. The contrast in OCT is due to the light scattering properties of cells, stroma, and other tissue structures and utilizes longer wavelengths in the near infrared region, which allows imaging at high resolutions deeper into tissues. The image is constructed by illuminating the sample and measuring the echo time delay and 
intensity of the backscattered light from the tissue. The tissue and boundaries causing the backscattered light have different optical properties and the dimensions of the tissue structure can be determined by measuring this backscattering time ${ }^{[359]}$. The mechanical components used for OCT can also be miniaturized, allowing them to be integrated into small probes, catheters, and endoscopes for imaging at internal sites ${ }^{[291]}$. In order to determine the longitudinal treatment response to nanotherapeutic delivery of rare ocular cancers, OCT was used and correlated with histopathology and flow cytometry, revealing relationships between neoplastic growth, neovascularization, and the immune microenvironment facilitating development of targeted therapies ${ }^{[360]}$. For investigating the TME, OCT has been used to investigate the collagen structures associated with bladder and skin cancer ${ }^{[361,362]}$ and to study the relationship between fibroblasts and mammary epithelial cells, in vitro, where the matrix stiffness was manipulated by changing the collagen content ${ }^{[363]}$. Assessment of the morphology of tumor vascular networks was performed using microstructural $\mathrm{OCT}^{[291]}$ and combining architectural and vascular OCT images acquired simultaneously allows for differentiating intra-tumor and peri-tumor vessels ${ }^{[364]}$. Other evaluations of vascular patterns have been performed in melanoma skin lesions with dynamic $\mathrm{OCT}^{[365]}$. The addition of contrast agents to OCT increases sensitivity, extending the capabilities into molecular imaging ${ }^{[366]}$ allowing for improved microvascular imaging ${ }^{[367-369]}$, investigation of the fundamental behaviors of tumor associated macrophages and other leukocytes through speckle-modulating $\mathrm{OCT}^{[370]}$ as well as the human epidermal growth factor receptor 2 (HER2 neu) protein with magnetomotive OCT which uses antibody conjugated magnetic nanoparticles ${ }^{[371]}$.

\section{CONCLUSION AND FUTURE WORK}

Factors within the TME are typically investigated individually in preclinical studies and the imaging modality most appropriate for that specific or desired TME target is used. However, the TME is highly variable and there are dynamic interactions between the TME and cancer cells that affect tumor growth, development, and response to nanotherapies ${ }^{[372]}$. Thus, there is an urgent need for improved analysis of interactions and crosstalk within the TME, which requires advanced imaging techniques. As described above, standard imaging modalities currently used in the clinic are CT, MRI, PET, SPECT, and ultrasound where there are both advantages and disadvantages to these techniques when evaluating the tumor microenvironment. MRI, PET, and SPECT are very expensive to use and have long acquisition times; however, they provide excellent sensitivity and soft tissue contrast. PET and SPECT also require exposure to radiation. CT is not as costly and allows rapid, whole-body imaging with high resolution but has low soft tissue contrast and similarly requires exposure to radiation. Ultrasound is only semiquantitative with low soft tissue contrast but has the advantages of being a real-time imaging method with rapid, high spatial, and temporal resolution along with low cost. Other advancements in ultrasound imaging, including the use of contrast agents and the development of photoacoustic imaging, have demonstrated greatly improved soft tissue contrast. The continued development of safe and effective contrast agents will permit improvements in all clinical imaging modalities ${ }^{[373]}$. Optical imaging methods including PAI, OCT, FMT, intravital microscopy, fluorescence, and bioluminescence have a much higher sensitivity for contrast agents and a much broader availability of probes permitting detection of multiple TME components, but are limited by imaging depth, small field of view, and difficulty in quantitation ${ }^{[374]}$. There is an inherent tradeoff between imaging resolution and depth of penetration and the optimal selected method will be dependent upon the desired imaging goal and target. A summary of the imaging methods discussed with their advantages in resolution and imaging depth as well as safety concerns can be found in Table 2 .

Currently, there is not one single imaging technology that can simply screen multiple TME factors in a single scan. Thus, to identify the dominant factor that alters tumor delivery of nanoparticles, multiple imaging technologies or varying contrast would need to be applied to each tumor in a similar fashion to immunohistochemical staining of tumor samples to evaluate a series of TME factors. In order to better select the appropriate imaging technique, it is important to know the advantages and disadvantages of the 


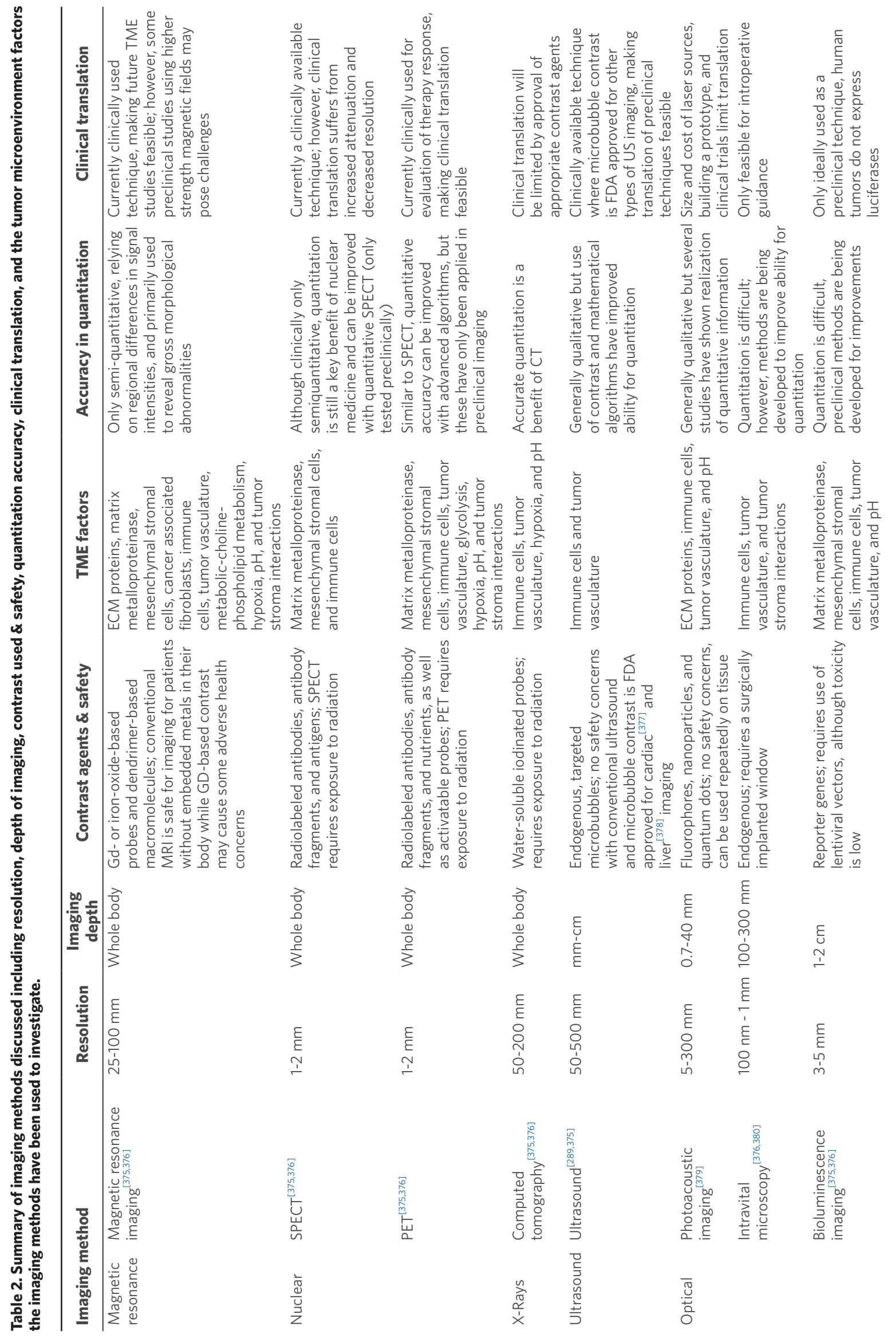




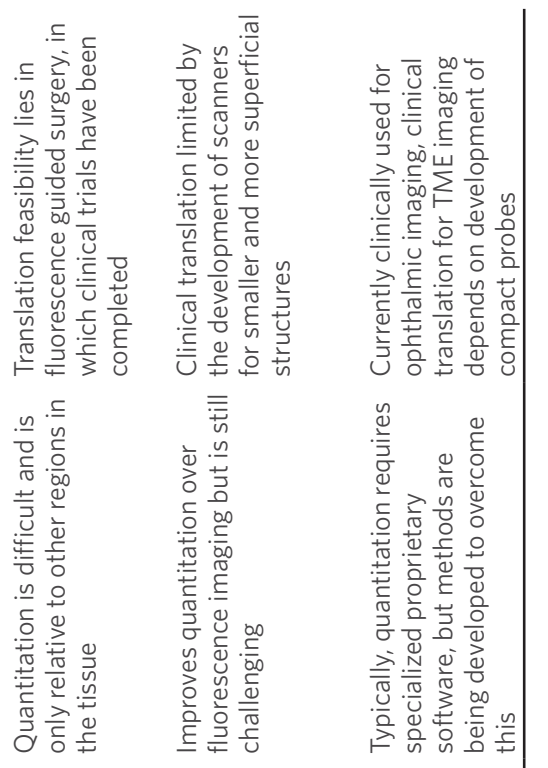

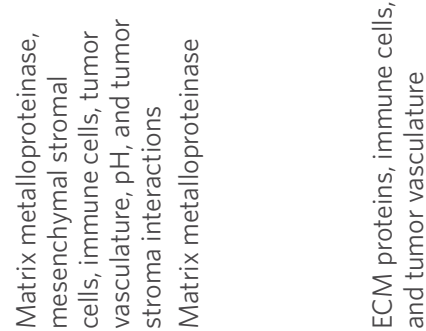
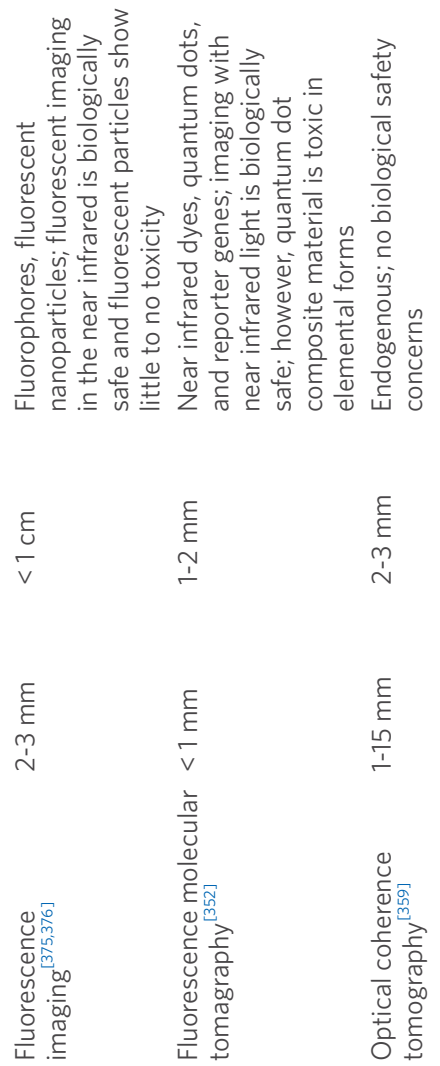

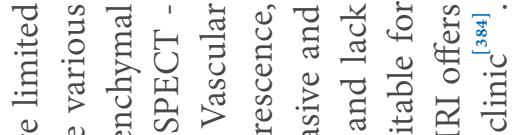

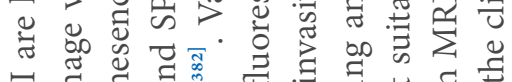

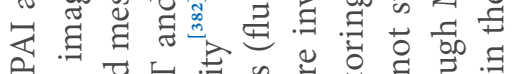

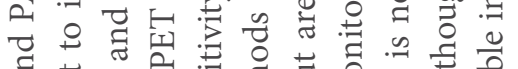

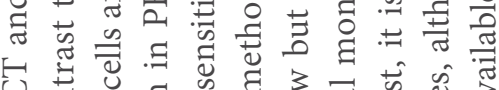

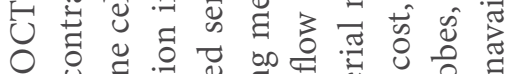

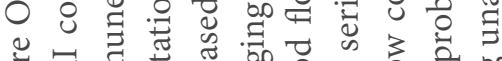

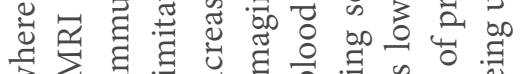

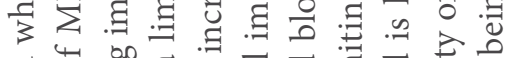
ర్屯

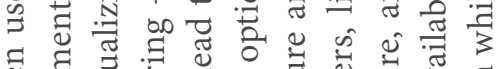
ปี

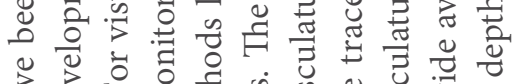

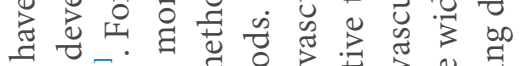

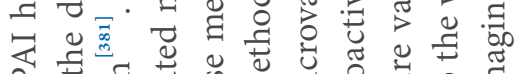

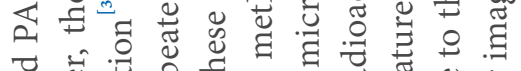

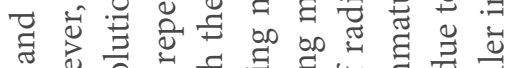

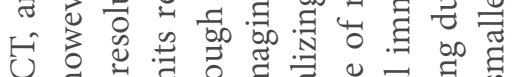

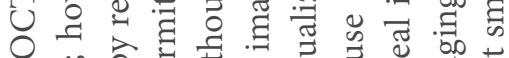

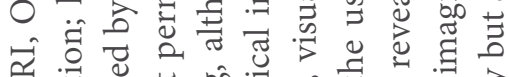

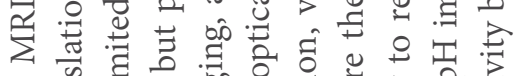

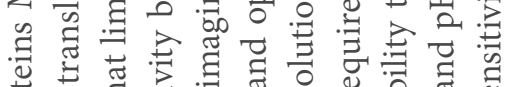

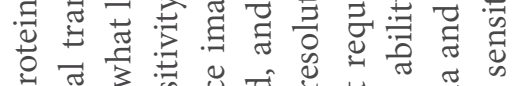

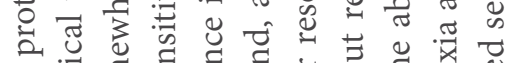

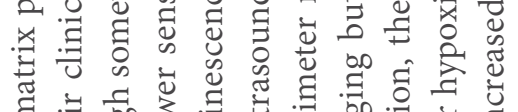

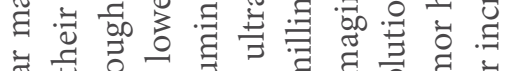

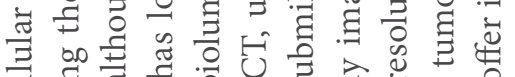

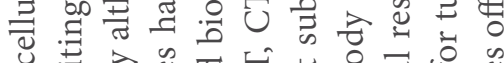

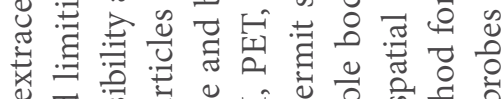

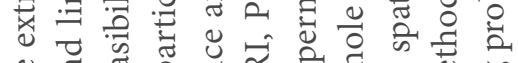

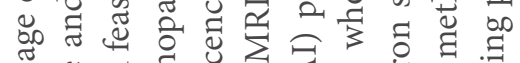

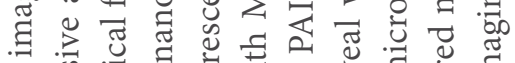

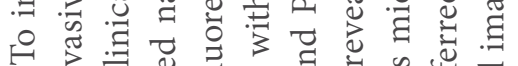

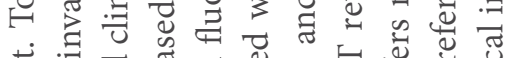

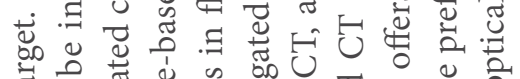

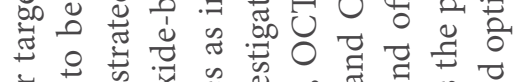

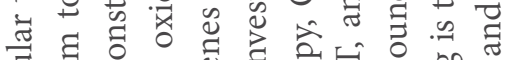

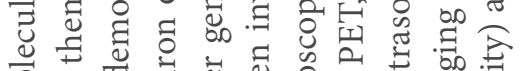
更

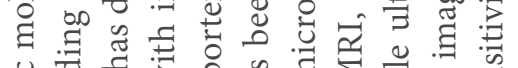

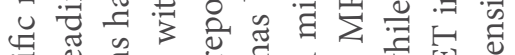

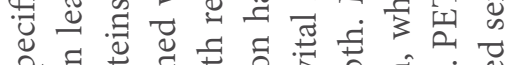
के घี त्ञ 范 ‡

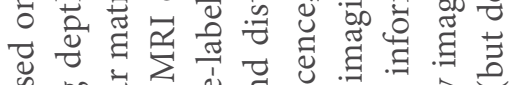

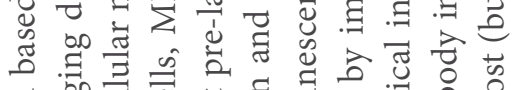
च

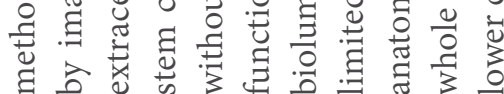

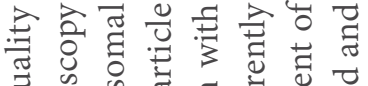
హ)

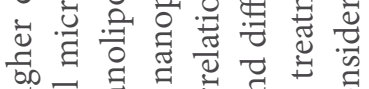

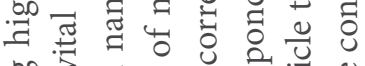
bo

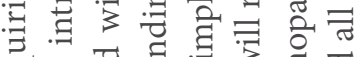

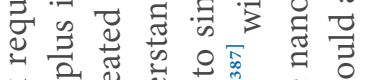

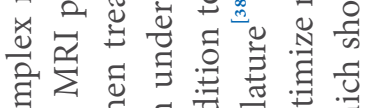

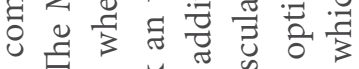

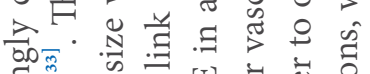

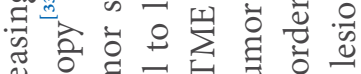

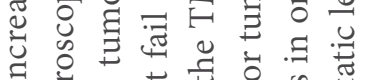
.

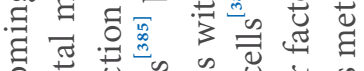

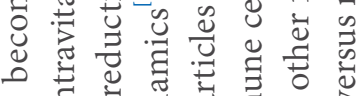

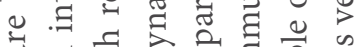

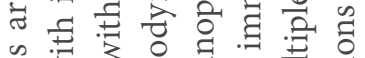

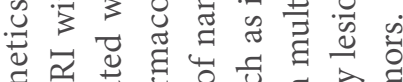

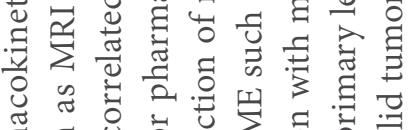

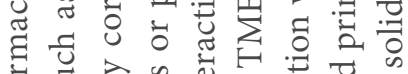

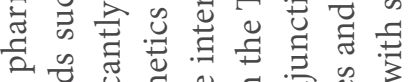

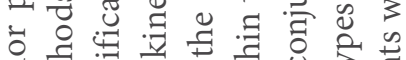

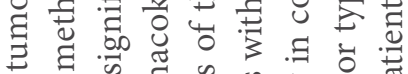
荡

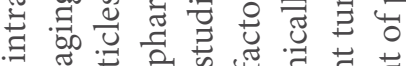

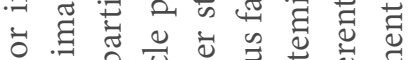

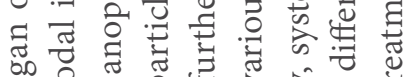

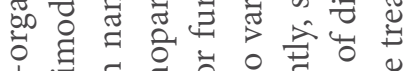
봃

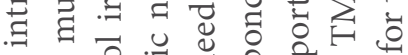

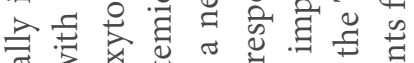
उัँ

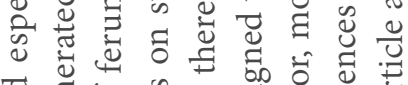

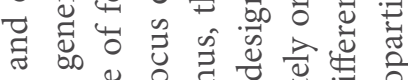

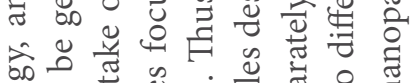

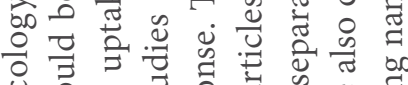

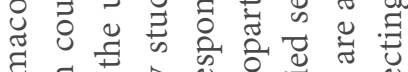

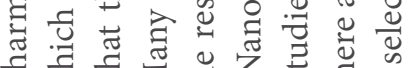

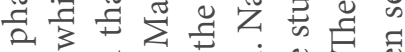

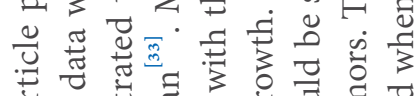

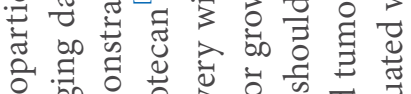

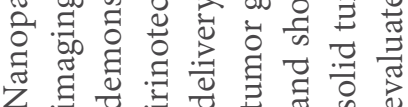




\section{DECLARATIONS}

\section{Acknowledgments}

The authors would like to acknowledge Dr. David Lalush, Associate Professor of Biomedical Engineering at the University of North Carolina and North Carolina State University and the Biomedical Research Imaging Center at the University of North Carolina, for his assistance editing. The authors would also like to acknowledge that the graphical abstract was created with BioRender.com.

\section{Authors' contributions}

Performed the literature search and wrote the manuscript: Moody AS

Edited all drafts, and reviewed the final draft: Dayton PA

Conceived the article, edited all drafts, and reviewed the final draft: Zamboni WC

\section{Availability of data and materials}

Not applicable.

\section{Financial support and sponsorship}

This work was supported by Grant name National Cancer Institute of the National Institutes of Health Postdoctoral Training Program Grant Number: T32CA196589.

\section{Conflicts of interest}

P.A.D. Declares inventorship on patents describing ultrasound imaging, contrast agents, and ultrasound therapeutics. He is a co-founder of SonoVol, Inc., and Triangle Biotechnology, Inc., companies which have licensed these patents.

\section{Ethical approval and consent to participate}

Not applicable.

\section{Consent for publication}

Not applicable.

\section{Copyright}

(c) The Author(s) 2021.

\section{REFERENCES}

1. Baghban R, Roshangar L, Jahanban-Esfahlan R, et al. Tumor microenvironment complexity and therapeutic implications at a glance. Cell Commun Signal 2020;18:59.

2. Król M, Pawłowski KM, Majchrzak K, Szyszko K, Motyl T. Why chemotherapy can fail? Pol J Vet Sci 2010;13:399-406.

3. Boyce MW, Labonia GJ, Hummon AB, Lockett MR. Assessing chemotherapeutic effectiveness using a paper-based tumor model. Analyst 2017;142:2819-27.

4. Jain RK, Munn LL, Fukumura D. Measuring vascular permeability in mice. Cold Spring Harb Protoc 2013;2013:444-6.

5. Madden AJ, Rawal S, Sandison K, et al. Evaluation of the efficiency of tumor and tissue delivery of carrier-mediated agents (CMA) and small molecule (SM) agents in mice using a novel pharmacokinetic (PK) metric: relative distribution index over time (RDI-OT). $J$ Nanoparticle Res 2014;16:2662.

6. Truong AS, Lockett MR. Oxygen as a chemoattractant: Confirming cellular hypoxia in paper-based invasion assays. Analyst 2016:141:3874-82.

7. Diop-Frimpong B, Chauhan VP, Krane S, Boucher Y, Jain RK. Losartan inhibits collagen I synthesis and improves the distribution and efficacy of nanotherapeutics in tumors. Proc Natl Acad Sci U S A 2011;108:2909-14.

8. Geevarghese A, Herman IM. Pericyte-endothelial crosstalk: Implications and opportunities for advanced cellular therapies.Transl Res 2014;163:296-306.

9. Stylianopoulos T, Jain RK. Combining two strategies to improve perfusion and drug delivery in solid tumors. Proc Natl Acad Sci U S A 2013;110:18632-7.

10. Zhang B, Hu Y, Pang Z. Modulating the tumor microenvironment to enhance tumor nanomedicine delivery. Front Pharmacol $2017 ; 8: 952$. 
11. Cairns R, Papandreou I, Denko N. Overcoming physiologic barriers to cancer treatment by molecularly targeting the tumor microenvironment. Mol Cancer Res 2006;4:61-70.

12. Jing X, Yang F, Shao C, et al. Role of hypoxia in cancer therapy by regulating the tumor microenvironment. Mol Cancer 2019;18:157.

13. Lucas AT, White TF, Deal AM, et al. Profiling the relationship between tumor-associated macrophages and pharmacokinetics of liposomal agents in preclinical murine models. Nanomedicine 2017;13:471-82.

14. Zamboni WC, Eiseman JL, Strychor S, et al. Tumor disposition of pegylated liposomal CKD-602 and the reticuloendothelial system in preclinical tumor models. J Liposome Res 2011;21:70-80.

15. Zhao CY, Cheng R, Yang Z, Tian ZM. Nanotechnology for cancer therapy based on chemotherapy. Molecules 2018;23:826.

16. Jain RK. Normalizing tumor microenvironment to treat cancer: Bench to bedside to biomarkers. J Clin Oncol 2013;31:2205-18.

17. Henke E, Nandigama R, Ergün S. Extracellular Matrix in the Tumor Microenvironment and Its Impact on Cancer Therapy. Front Mol Biosci 2020;6:160.

18. Gonzalez-Avila G, Sommer B, Mendoza-Posada DA, Ramos C, Garcia-Hernandez AA, Falfan-Valencia R. Matrix metalloproteinases participation in the metastatic process and their diagnostic and therapeutic applications in cancer. Crit Rev Oncol Hematol 2019;137:5783.

19. Nwabo KAH, Kamga PT, Simo RT, et al. Mesenchymal stromal cells' role in tumor microenvironment: involvement of signaling pathways. Cancer Biol Med 2017;14:129-41.

20. Liu T, Zhou L, Li D, Andl T, Zhang Y. Cancer-associated fibroblasts build and secure the tumor microenvironment. Front Cell Dev Biol 2019;7:60

21. Yoshida GJ. Regulation of heterogeneous cancer-associated fibroblasts: The molecular pathology of activated signaling pathways. $J$ Exp Clin Cancer Res 2020;39:112.

22. Yin Y, Yao S, Hu Y, et al. The immune-microenvironment confers chemoresistance of colorectal cancer through macrophage-derived IL6. Clin Cancer Res 2017;23:7375-87.

23. Castells M, Thibault B, Delord JP, Couderc B. Implication of tumor microenvironment in chemoresistance: Tumor-associated stromal cells protect tumor cells from cell death. Int J Mol Sci 2012;13:9545-71.

24. Senthebane DA, Rowe A, Thomford NE, et al. The role of tumor microenvironment in chemoresistance: To survive, keep your enemies closer. Int J Mol Sci 2017;18:1586.

25. Bagnoli M, Granata A, Nicoletti R, et al. Choline metabolism alteration: A focus on ovarian cancer. Front Oncol 2016;6:153.

26. Saggar JK, Yu M, Tan Q, Tannock IF. The tumor microenvironment and strategies to improve drug distribution. Front Oncol 2013;3:154.

27. Zhong S, Jeong JH, Chen Z, Chen Z, Luo JL. Targeting tumor microenvironment by small-molecule inhibitors.Transl Oncol 2020;13:5769.

28. Correia AL, Bissell MJ. The tumor microenvironment is a dominant force in multidrug resistance. Drug Resist Updat 2012;15:39-49.

29. Graham K, Unger E. Overcoming tumor hypoxia as a barrier to radiotherapy, chemotherapy and immunotherapy in cancer treatment. Int J Nanomedicine 2018;13:6049-58.

30. Tian Y, Zhang Y, Teng Z, et al. pH-dependent transmembrane activity of peptide-functionalized gold nanostars for computed tomography/ photoacoustic imaging and photothermal therapy. ACS Appl Mater Interfaces 2017;9:2114-22.

31. Liu H, Wang H, Xu Y, et al. Lactobionic acid-modified dendrimer-entrapped gold nanoparticles for targeted computed tomography imaging of human hepatocellular carcinoma. ACS Appl Mater Interfaces 2014;6:6944-53.

32. Kim Y, Oh Y-H, Oh E, Kim H-S. Chip-based protease assay using fluorescence resonance energy transfer between quantum dots and fluorophores. Biochip J 2007;1:228-33.

33. Ramanathan RK, Korn RL, Raghunand N, et al. Correlation between ferumoxytol uptake in tumor lesions by MRI and response to nanoliposomal irinotecan in patients with advanced solid tumors: A pilot study. Clin Cancer Res 2017;23:363848.

34. Cuccarese MF, Dubach JM, Pfirschke C, et al. Heterogeneity of macrophage infiltration and therapeutic response in lung carcinoma revealed by 3D organ imaging. Nat Commun 2017;8:14293.

35. Banerjee SR, Foss CA, Horhota A, et al. ${ }^{111}$ In- and IRDye800CW-Labeled PLA-PEG Nanoparticle for Imaging Prostate-Specific Membrane Antigen-Expressing Tissues. Biomacromolecules 2017;18:201-9.

36. Lee H, Shields AF, Siegel BA, et al. 64Cu-MM-302 positron emission tomography quantifies variability of enhanced permeability and retention of nanoparticles in relation to treatment response in patients with metastatic breast cancer. Clin Cancer Res 2017;23:4190-202.

37. Rojas JD, Papadopoulou V, Czernuszewicz TJ, et al. Ultrasound Measurement of Vascular Density to Evaluate Response to AntiAngiogenic Therapy in Renal Cell Carcinoma. IEEE Trans Biomed Eng 2019;66:873-80.

38. Du J, Lane LA, Nie S. Stimuli-responsive nanoparticles for targeting the tumor microenvironment. J Control Release 2015;219:205-14.

39. Yin C, Wen G, Liu C, et al. Organic Semiconducting Polymer Nanoparticles for Photoacoustic Labeling and Tracking of Stem Cells in the Second Near-Infrared Window. ACS Nano 2018;12:12201-11.

40. Wu X, Hu J, Zhou L, et al. In vivo tracking of superparamagnetic iron oxide nanoparticle-labeled mesenchymal stem cell tropism to malignant gliomas using magnetic resonance imaging: Laboratory investigation. J Neurosurg 2008;108:320-9.

41. Karageorgis A, Dufort S, Sancey L, et al. An MRI-based classification scheme to predict passive access of 5 to 50-nm large nanoparticles to tumors. Sci Rep 2016;6:21417.

42. Miller MA, Gadde S, Pfirschke C, et al. Predicting therapeutic nanomedicine efficacy using a companion magnetic resonance imaging nanoparticle. Sci Transl Med 2015;7:314ra183.

43. Hauge A, Wegner CS, Gaustad JV, Simonsen TG, Andersen LMK, Rofstad EK. Diffusion-weighted MRI-derived ADC values reflect collagen I content in PDX models of uterine cervical cancer. Oncotarget 2017;8:105682-91. 
44. Wahyudi H, Reynolds AA, Li Y, Owen SC, Yu SM. Targeting collagen for diagnostic imaging and therapeutic delivery. J Control Release 2016;240:323-31.

45. Shiftan L, Israely T, Cohen M, et al. Magnetic resonance imaging visualization of hyaluronidase in ovarian carcinoma. Cancer Res 2005;65:10316-23.

46. Guo C, Sun L, Cai H, et al. Gadolinium-Labeled Biodegradable Dendron-Hyaluronic Acid Hybrid and Its Subsequent Application as a Safe and Efficient Magnetic Resonance Imaging Contrast Agent. ACS Appl Mater Interfaces 2017;9:23508-19.

47. Granot D, Addadi Y, Kalchenko V, Harmelin A, Kunz-Schughart LA, Neeman M. In vivo imaging of the systemic recruitment of fibroblasts to the angiogenic rim of ovarian carcinoma tumors. Cancer Res 2007;67:9180-9.

48. Granot D, Kunz-Schughart LA, Neeman M. Labeling fibroblasts with biotin-BSA-GdDTPA-FAM for tracking of tumor-associated stroma by fluorescence and MR imaging. Magn Reson Med 2005;54:789-97.

49. Reagan MR, Kaplan DL. Concise review: Mesenchymal stem cell tumor-homing: Detection methods in disease model systems. Stem Cells 2011;29:920-7.

50. Loebinger MR, Kyrtatos PG, Turmaine M, et al. Magnetic resonance imaging of mesenchymal stem cells homing to pulmonary metastases using biocompatible magnetic nanoparticles. Cancer Res 2009;69:8862-7.

51. Belmar-Lopez C, Mendoza G, Oberg D, et al. Tissue-derived mesenchymal stromal cells used as vehicles for anti-tumor therapy exert different in vivo effects on migration capacity and tumor growth. BMC Med 2013;11:139.

52. Lewis CE, Pollard JW. Distinct role of macrophages in different tumor microenvironments. Cancer Res 2006;66:605-12.

53. Zolnik BS, González-Fernández Á, Sadrieh N, Dobrovolskaia MA. Minireview: Nanoparticles and the immune system. Endocrinology 2010;2:458-65.

54. Song G, Wu H, Yoshino K, Zamboni WC. Factors affecting the pharmacokinetics and pharmacodynamics of liposomal drugs. J Liposome Res 2012 Sep;22:177-92.

55. Daldrup-Link HE, Golovko D, Ruffell B, et al. MRI of tumor-associated macrophages with clinically applicable iron oxide nanoparticles. Clin Cancer Res 2011;17:5695-704.

56. Reichel D, Tripathi M, Perez JM. Biological effects of nanoparticles on macrophage polarization in the tumor microenvironment. Nanotheranostics 2019;3:66-88.

57. Harisinghani MG, Barentsz J, Hahn PF, et al. Noninvasive detection of clinically occult lymph-node metastases in prostate cancer. $N$ Engl J Med 2003;348:2491-9.

58. Harisinghani MG, Weissleder R. Sensitive, noninvasive detection of lymph node metastases. PLoS Med 2004;1:e66.

59. Heesakkers RAM, Jager GJ, Hövels AM, et al. Prostate cancer: Detection of lymph node metastases outside the routine surgical area with ferumoxtran-10-enhanced MR imaging. Radiology 2009;251:408-14.

60. Lepage M, Dow WC, Melchior M, et al. Noninvasive detection of matrix metalloproteinase activity in vivo using a novel magnetic resonance imaging contrast agent with a solubility switch. Mol Imaging 2007;6:393-403.

61. Lebel R, Jastrzębska B, Therriault H, et al. Novel solubility-switchable MRI agent allows the noninvasive detection of matrix metalloproteinase-2 activity in vivo in a mouse model. Magn Reson Med 2008;60:1056-65.

62. Lebel R, Lepage M. A comprehensive review on controls in molecular imaging: Lessons from MMP-2 imaging. Contrast Media Mol Imaging 2014;9:187-210.

63. Ansari C, Tikhomirov GA, Hong SH, et al. Development of novel tumor-targeted theranostic nanoparticles activated by membrane-type matrix metalloproteinases for combined cancer magnetic resonance imaging and therapy. Small 2014;10:566-75,417.

64. Matsuo K, Kamada R, Mizusawa K, et al. Specific detection and imaging of enzyme activity by signal-amplifiable self-assembling $19 \mathrm{Fa}$ MRI probes. Chemistry 2013;19:12875-83.

65. Crisp JL, Savariar EN, Glasgow HL, Ellies LG, Whitney MA, Tsien RY. Dual targeting of integrin $\alpha v \beta 3$ and matrix metalloproteinase-2 for optical imaging of tumors and chemotherapeutic delivery. Mol Cancer Ther 2014;13:1514-25.

66. Kawamura W, Miura Y, Kokuryo D, et al. Density-tunable conjugation of cyclic RGD ligands with polyion complex vesicles for the neovascular imaging of orthotopic glioblastomas. Sci Technol Adv Mater 2015;16:035004.

67. Yan C, Wu Y, Feng J, et al. Anti- $\alpha \mathrm{v} \beta 3$ antibody guided three-step pretargeting approach using magnetoliposomes for molecular magnetic resonance imaging of breast cancer angiogenesis. Int J Nanomedicine 2013;8:245-55.

68. Barrett T, Brechbiel M, Bernardo M, Choyke PL. MRI of tumor angiogenesis. J Magn Reson Imaging 2007;26:235-49.

69. Willats L, Calamante F. The 39 steps: Evading error and deciphering the secrets for accurate dynamic susceptibility contrast MRI. NMR Biomed 2013;26:913-31.

70. Morita N, Wang S, Chawla S, Poptani H, Melhem ER. Dynamic susceptibility contrast perfusion weighted imaging in grading of nonenhancing astrocytomas. J Magn Reson Imaging 2010;32:803-8.

71. Chakhoyan A, Raymond C, Chen J, et al. Probabilistic independent component analysis of dynamic susceptibility contrast perfusion MRI in metastatic brain tumors. Cancer Imaging 2019;19:14.

72. Barnes SL, Whisenant JG, Loveless ME, Yankeelov TE. Practical Dynamic Contrast Enhanced MRI in Small Animal Models of Cancer: Data Acquisition, Data Analysis, and Interpretation. Pharmaceutics 2012;4:442-78.

73. Brix G, Griebel J, Kiessling F, Wenz F. Tracer kinetic modelling of tumour angiogenesis based on dynamic contrast-enhanced CT and MRI measurements. Eur J Nucl Med Mol Imaging 2010;37 Suppl 1:S30-51.

74. Koh TS, Bisdas S, Koh DM, Thng CH. Fundamentals of tracer kinetics for dynamic contrast-enhanced MRI. J Magn Reson Imaging 2011;34:1262-76.

75. O’Connor JPB, Rose CJ, Waterton JC, Carano RAD, Parker GJM, Jackson A. Imaging intratumor heterogeneity: Role in therapy 
response, resistance, and clinical outcome. Clin Cancer Res 2015;21:249-57.

76. Koh DM, Collins DJ, Orton MR. Intravoxel incoherent motion in body diffusion-weighted MRI: Reality and challenges. $A J R A m J$ Roentgenol 2011 Jun;196:1351-61.

77. Pathak AP, Penet MF, Bhujwalla ZM. MR Molecular Imaging of Tumor Vasculature and Vascular Targets. Adv Genet 2010;69:1-30.

78. Abramovitch R, Dafni H, Smouha E, Benjamin LE, Neeman M. In vivo prediction of vascular susceptibility to vascular endothelial growth factor withdrawal: Magnetic resonance imaging of C6 rat glioma in nude mice. Cancer Res 1999;59:5012-6.

79. Carmeliet P, Dor Y, Herber JM, et al. Role of HIF-1 $\alpha$ in hypoxiamediated apoptosis, cell proliferation and tumour angiogenesis. Nature 1998;394:485-90.

80. O'Connor JPB, Boult JKR, Jamin Y, et al. Oxygen-enhanced MRI accurately identifies, quantifies, and maps tumor hypoxia in preclinical cancer models. Cancer Res 2016;76:787-95.

81. O'Connor JPB, Robinson SP, Waterton JC. Imaging tumour hypoxia with oxygen-enhanced MRI and BOLD MRI. Br J Radiol 2019;92:20180642.

82. White DA, Zhang Z, Li L, et al. Developing oxygen-enhanced magnetic resonance imaging as a prognostic biomarker of radiation response. Cancer Lett 2016;380:69-77.

83. Zhao D, Jiang L, Hahn EW, Mason RP. Comparison of1H blood oxygen level-dependent (BOLD) and 19F MRI to investigate tumor oxygenation. Magn Reson Med 2009;62:357-64.

84. Lin YC, Wang JJ, Hong JH, et al. Noninvasive monitoring of microvascular changes with partial irradiation using dynamic contrastenhanced and blood oxygen level-dependent magnetic resonance imaging. Int J Radiat Oncol Biol Phys 2013;85:1367-74.

85. Stoyanova R, Huang K, Sandler K, et al. Mapping tumor hypoxia in vivo using pattern recognition of dynamic contrast-enhanced MRI data. Transl Oncol 2012;5:437-47.

86. Egeland TAM, Gulliksrud K, Gaustad JV, Mathiesen B, Rofstad EK. Dynamic contrast-enhanced-MRI of tumor hypoxia. Magn Reson Med 2012;67:519-30.

87. Gulliksrud K, Øvrebø KM, Mathiesen B, Rofstad EK. Differentiation between hypoxic and non-hypoxic experimental tumors by dynamic contrast-enhanced magnetic resonance imaging. Radiother Oncol 2011;98:360-4.

88. Wojtkowiak JW, Verduzco D, Schramm KJ, Gillies RJ. Drug resistance and cellular adaptation to tumor acidic $\mathrm{pH}$ microenvironment. Mol Pharm 2011;8:2032-8.

89. Yamamoto K, Brender JR, Seki T, et al. Molecular Imaging of the Tumor Microenvironment Reveals the Relationship between Tumor Oxygenation, Glucose Uptake, and Glycolysis in Pancreatic Ductal Adenocarcinoma. Cancer Res 2020;80:2087-93.

90. Rodrigues TB, Serrao EM, Kennedy BWC, Hu DE, Kettunen MI, Brindle KM. Magnetic resonance imaging of tumor glycolysis using hyperpolarized 13 C-labeled glucose. Nat Med 2014;20:93-7.

91. Gallagher FA, Kettunen MI, Day SE, et al. Magnetic resonance imaging of $\mathrm{pH}$ in vivo using hyperpolarized 13C-labelled bicarbonate. Nature 2008;453:940-3.

92. Scholz DJ, Janich MA, Köllisch U, et al. Quantified pH imaging with hyperpolarized 13C-bicarbonate. Magn Reson Med 2015;73:227482.

93. Ward KM, Balaban RS. Determination of $\mathrm{pH}$ using water protons and Chemical Exchange Dependent Saturation Transfer (CEST). Magn Reson Med 2000 Nov;44:799-802.

94. Huang Y, Coman D, Ali MM, Hyder F. Lanthanide ion (III) complexes of 1,4,7,10-tetraazacyclododecane-1,4,7,10-tetraaminophosphonate for dual biosensing of $\mathrm{pH}$ with chemical exchange saturation transfer (CEST) and biosensor imaging of redundant deviation in shifts (BIRDS). Contrast Media Mol Imaging 2015;10:51-8.

95. Chen LQ, Randtke EA, Jones KM, Moon BF, Howison CM, Pagel MD. Evaluations of tumor acidosis within in vivo tumor models using parametric maps generated with AcidoCEST MRI. Mol Imaging Biol 2015;17:488-96.

96. Longo DL, Sun PZ, Consolino L, Michelotti FC, Uggeri F, Aime S. A general MRI-CEST ratiometric approach for pH imaging: Demonstration of in vivo pH mapping with iobitridol. J Am Chem Soc 2014;136:14333-6.

97. Aime S, Delli Castelli D, Terreno E. Novel pH-reporter MRI contrast agents. Angew Chem Int Ed Engl 2002;41:4334-6.

98. Sheth VR, Li Y, Chen LQ, Howison CM, Flask CA, Pagel MD. Measuring in vivo tumor pHe with CEST-FISP MRI. Magn Reson Med 2012;67:760-8.

99. Chen Y, Yin Q, Ji X, et al. Manganese oxide-based multifunctionalized mesoporous silica nanoparticles for pH-responsive MRI, ultrasonography and circumvention of MDR in cancer cells. Biomaterials 2012;33:7126-37.

100. Dixon WT, Ren J, Lubag AJM, et al. A concentration-independent method to measure exchange rates in PARACEST agents. Magn Reson Med 2010;63:625-32.

101. Marathe K, McVicar N, Li A, Bellyou M, Meakin S, Bartha R. Topiramate induces acute intracellular acidification in glioblastoma. $J$ Neurooncol 2016;130:465-72.

102. Albatany M, Li A, Meakin S, Bartha R. Dichloroacetate induced intracellular acidification in glioblastoma: in vivo detection using AACID-CEST MRI at 9.4 Tesla. J Neurooncol 2018;136:255-62.

103. Albatany M, Li A, Meakin S, Bartha R. In vivo detection of acute intracellular acidification in glioblastoma multiforme following a single dose of cariporide. Int J Clin Oncol 2018;23:812-9.

104. Albatany M, Ostapchenko VG, Meakin S, Bartha R. Brain tumor acidification using drugs simultaneously targeting multiple $\mathrm{pH}$ regulatory mechanisms. J Neurooncol 2019;144:453-62.

105. Harris RJ, Cloughesy TF, Liau LM, et al. PH-weighted molecular imaging of gliomas using amine chemical exchange saturation transfer MRI. Neuro Oncol 2015; 17:1514-24. 
106. Yao J, Tan CHP, Schlossman J, et al. pH-weighted amine chemical exchange saturation transfer echoplanar imaging (CEST-EPI) as a potential early biomarker for bevacizumab failure in recurrent glioblastoma. $J$ Neurooncol 2019;142:587-95.

107. Longo DL, Michelotti F, Consolino L, et al. In vitro and in vivo assessment of nonionic iodinated radiographic molecules as chemical exchange saturation transfer magnetic resonance imaging tumor perfusion agents. Invest Radiol 2016;51:155-62.

108. Longo DL, Bartoli A, Consolino L, et al. In vivo imaging of tumor metabolism and acidosis by combining PET and MRI-CEST pH imaging. Cancer Res 2016;76:6463-70.

109. Anemone A, Consolino L, Conti L, et al. In vivo evaluation of tumour acidosis for assessing the early metabolic response and onset of resistance to dichloroacetate by using magnetic resonance pH imaging. Int J Oncol 2017;51:498-506.

110. Aime S, Calabi L, Biondi L, et al. Iopamidol: Exploring the potential use of a well-established x-ray contrast agent for MRI. Magn Reson Med 2005;53:830-4.

111. Anemone A, Consolino L, Longo DL. MRI-CEST assessment of tumour perfusion using X-ray iodinated agents: comparison with a conventional Gd-based agent. Eur Radiol 2017;27:2170-9.

112. Jones KM, Randtke EA, Yoshimaru ES, et al. Clinical Translation of Tumor Acidosis Measurements with AcidoCEST MRI. Mol Imaging Biol 2017;19:617-25.

113. Sun PZ, Longo DL, Hu W, Xiao G, Wu R. Quantification of iopamidol multi-site chemical exchange properties for ratiometric chemical exchange saturation transfer (CEST) imaging of pH. Phys Med Biol 2014;59:4493-504.

114. Jones KM, Randtke EA, Howison CM, Pagel MD. Respiration gating and Bloch fitting improve pH measurements with acidoCEST MRI in an ovarian orthotopic tumor model. Proc SPIE Int Soc Opt Eng 2016;9788:978815.

115. Randtke EA, Granados JC, Howison CM, Pagel MD, Cárdenas-Rodríguez J. Multislice CEST MRI improves the spatial assessment of tumor pH. Magn Reson Med 2017;78:97-106.

116. Wu R, Longo DL, Aime S, Sun PZ. Quantitative description of radiofrequency (RF) power-based ratiometric chemical exchange saturation transfer (CEST) pH imaging. NMR Biomed 2015;28:555-65.

117. Arena F, Irrera P, Consolino L, Colombo Serra S, Zaiss M, Longo DL. Flip-angle based ratiometric approach for pulsed CEST-MRI pH imaging. J Magn Reson 2018;287:1-9.

118. Khalil MM, Tremoleda JL, Bayomy TB, Gsell W. Molecular SPECT Imaging: An Overview. Int J Mol Imaging 2011;2011:796025.

119. Phelps ME. PET: The merging of biology and imaging into molecular imaging. J Nucl Med 2000;41:661-81.

120. Chen K, Chen X. Design and Development of Molecular Imaging Probes. Curr Top Med Chem 2010;10:1227-36.

121. Niu G, Chen X. PET Imaging of Angiogenesis. PET Clinics 2009;4:17-38.

122. Scherer RL, McIntyre JO, Matrisian LM. Imaging matrix metalloproteinases in cancer. Cancer Metastasis Rev 2008;27:679-90.

123. Dwyer RM, Ryan J, Havelin RJ, et al. Mesenchymal stem cell-mediated delivery of the sodium iodide symporter supports radionuclide imaging and treatment of breast cancer. Stem Cells 2011;29:1149-57.

124. Sakr TM, Essa BM, El-Essawy FA, El-Mohty AA. Synthesis and biodistribution of 99m Tc-PyDA as a potential marker for tumor hypoxia imaging. Radiochemistry 2014;56:76-80.

125. Juergens RA, Zukotynski KA, Singnurkar A, Snider DP, Valliant JF, Gulenchyn KY. Imaging Biomarkers in Immunotherapy. Biomark Cancer 2016;8:1-13.

126. Man F, Lammers T, T. M. de Rosales R. Imaging Nanomedicine-Based Drug Delivery: a Review of Clinical Studies. Mol Imaging Biol 2018 Oct;20:683-95.

127. Koukourakis MI, Koukouraki S, Giatromanolaki A, et al. Liposomal doxorubicin and conventionally fractionated radiotherapy in the treatment of locally advanced non-small-cell lung cancer and head and neck cancer. J Clin Oncol 1999;17:3512-21.

128. Koukourakis MI, Koukouraki S, Giatromanolaki A, et al. High intratumoral accumulation of stealth liposomal doxorubicin in sarcomas Rationale for combination with radiotherapy. Acta Oncol 2000;39:207-11.

129. Pérez-Medina C, Abdel-Atti D, Tang J, et al. Nanoreporter PET predicts the efficacy of anti-cancer nanotherapy. Nat Commun 2016;7:11838.

130. Matusiak N, Castelli R, Tuin AW, et al. A dual inhibitor of matrix metalloproteinases and a disintegrin and metalloproteinases, $\left[{ }^{18} \mathrm{~F}\right] \mathrm{FB}-$ ML5, as a molecular probe for non-invasive MMP/ADAM-targeted imaging. Bioorganic Med Chem 2015;23:192-202.

131. Altiparmak B, Lambrecht FY, Citak A. Design of radiolabeled gelatinase inhibitor peptide (99mTc-CLP) and evaluation in rats. Appl Radiat Isot 2014;89:130-3.

132. Da Rocha Gomes S, Miguel J, Azéma L, et al. 99mTc-MAG3-aptamer for imaging human tumors associated with high level of matrix metalloprotease-9. Bioconjug Chem 2012;23:2192-200.

133. Kondo N, Temma T, Shimizu Y, Watanabe H, Higano K, Takagi Y, et al. Miniaturized antibodies for imaging membrane type-1 matrix metalloproteinase in cancers. Cancer Sci 2013;104:495-501.

134. van Duijnhoven SMJ, Robillard MS, Nicolay K, Grüll H. In vivo biodistribution of radiolabeled MMP-2/9 activatable cell-penetrating peptide probes in tumor-bearing mice. Contrast Media Mol Imaging 2015;10:59-66.

135. Huang T, Civelek AC, Zheng H, et al. (18)F-misonidazole PET imaging of hypoxia in micrometastases and macroscopic xenografts of human non-small cell lung cancer: a correlation with autoradiography and histological findings. Am J Nucl Med Mol Imaging 2013;3:14253.

136. Knoop K, Kolokythas M, Klutz K, et al. Image-guided, tumor stroma-targeted 131 i therapy of hepatocellular cancer after systemic mesenchymal stem cell-mediated nis gene delivery. Mol Ther 2011;19:1704-13.

137. Wolfs E, Struys T, Notelaers T, et al. 18F-FDG labeling of mesenchymal stem cells and multipotent adult progenitor cells for PET imaging: Effects on ultrastructure and differentiation capacity. J Nucl Med 2013;54:447-54. 
138. Patrick PS, Kolluri KK, Zaw Thin M, et al. Lung delivery of MSCs expressing anti-cancer protein TRAIL visualised with 89Zr-oxine PET-CT. Stem Cell Res Ther 2020;11:256.

139. Locke LW, Mayo MW, Yoo AD, Williams MB, Berr SS. PET imaging of tumor associated macrophages using mannose coated $64 \mathrm{Cu}$ liposomes. Biomaterials 2012;33:7785-93.

140. Rashidian M, Keliher EJ, Bilate AM, et al. Noninvasive imaging of immune responses. Proc Natl Acad Sci U S A 2015;112:6146-51.

141. Tavaré R, McCracken MN, Zettlitz KA, Knowles SM, Salazar FB, Olafsen T, et al. Engineered antibody fragments for immuno-PET imaging of endogenous CD8 + T cells in vivo. Proc Natl Acad Sci U S A 2014;111:1108-13.

142. Chotipanich C, Promteangtrong C, Kunawudhi A, et al. (11)C-Choline and FDG PET/CT Imaging of Primary Cholangiocarcinoma: A Comparative Analysis. Asia Ocean J Nucl Med Biol 2015;3:18-25.

143. Jadvar H. Prostate cancer: PET with 18F-FDG, 18F- or 11C-acetate, and 18F- or 11C-choline. J Nucl Med 2011;52:81-9.

144. Kryza D, Tadino V, Filannino MA, Villeret G, Lemoucheux L. Fully automated [18F]fluorocholine synthesis in the TracerLab MXFDG Coincidence synthesizer. Nucl Med Biol 2008;35:255-60.

145. Kumar R, Zhuang H, Alavi A. PET in the management of urologic malignancies. Radiol Clin North Am 2004;42:1141-53, ix.

146. Laking GR, West C, Buckley DL, Matthews J, Price PM. Imaging vascular physiology to monitor cancer treatment. Crit Rev Oncol Hematol 2006;58:95-113.

147. Wilson RA, Shea MJ, de Landsheere CM, et al. Validation of quantitation of regional myocardial blood flow in vivo with 11C-labeled human albumin microspheres and positron emission tomography. Circulation 1984;70:717-23.

148. Mullani NA, Herbst RS, Abbruzzese JL, Barron B, Lamki L, Charnsangavej C, et al. First pass FDG measured blood flow in tumors: A comparison with O-15 labeled water measured blood flow. Clin Positron Imaging 2000;3:153.

149. Laking GR, Price PM. Positron emission tomographic imaging of angiogenesis and vascular function. Br J Radiol 2003;76 Spec No 1:S50-9.

150. Hoffend J, Mier W, Schuhmacher J, et al. Gallium-68-DOTA-albumin as a PET blood-pool marker: Experimental evaluation in vivo. Nucl Med Biol 2005;32:287-92.

151. Kimura RH, Wang L, Shen B, et al. Evaluation of integrin $\alpha v \beta 6$ cystine knot PET tracers to detect cancer and idiopathic pulmonary fibrosis. Nat Commun 2019;10:4673.

152. Flechsig P, Lindner T, Loktev A, et al. PET/CT Imaging of NSCLC with a $\alpha$ v $\beta 6$ Integrin-Targeting Peptide. Mol Imaging Biol 2019;21:973-83.

153. Notni J, Reich D, Maltsev OV, et al. In Vivo PET imaging of the cancer integrin $\alpha v \beta 6$ using 68Ga-labeled cyclic RGD nonapeptides. $J$ Nucl Med 2017;58:671-7.

154. Beer AJ, Grosu AL, Carlsen J, et al. [18F]Galacto-RGD positron emission tomography for imaging of $\alpha \mathrm{v} \beta 3$ expression on the neovasculature in patients with squamous cell carcinoma of the head and neck. Clin Cancer Res 2007;13:6610-6.

155. Beer AJ, Haubner R, Sarbia M, et al. Positron emission tomography using [18F]Galacto-RGD identifies the level of integrin $\alpha \mathrm{v} \beta 3$ expression in man. Clin Cancer Res 2006;

156. Haubner R, Wester HJ, Burkhart F, et al. Glycosylated RGD-containing peptides: Tracer for tumor targeting and angiogenesis imaging with improved biokinetics. $J$ Nucl Med 2001;42:326-36.

157. Chen K, Cai W, Li ZB, Wang H, Chen X. Quantitative PET imaging of VEGF receptor expression. Mol Imaging Biol 2009;11:15-22.

158. Zhang Y, Hong H, Cai W. PET tracers based on Zirconium-89. Curr Radiopharm 2011;4:131-9.

159. Levashova Z, Backer M V., Horng G, Felsher D, Backer JM, Blankenberg FG. SPECT and PET imaging of EGF receptors with sitespecifically labeled EGF and dimeric EGF. Bioconjug Chem 2009;20:742-9.

160. Nagengast WB, De Vries EG, Hospers GA, et al. In vivo VEGF imaging with radiolabeled bevacizumab in a human ovarian tumor xenograft. $J$ Nucl Med 2007;48:1313-9.

161. Collingridge DR, Carroll VA, Glaser M, et al. The development of [124I]iodinated-VG76e: A novel tracer for imaging vascular endothelial growth factor in vivo using positron emission tomography. Cancer Res 2002;62:5912-9.

162. Scheer MGW, Stollman TH, Boerman OC, et al. Imaging liver metastases of colorectal cancer patients with radiolabelled bevacizumab: Lack of correlation with VEGF-A expression. Eur J Cancer 2008;44:1835-40.

163. Strauss LG, Koczan D, Klippel S, et al. Impact of angiogenesis-related gene expression on the tracer kinetics of $18 \mathrm{~F}-\mathrm{FDG}$ in colorectal tumors. J Nucl Med 2008;49:1238-44.

164. Cai W, Chen K, Mohamedali KA, et al. PET of vascular endothelial growth factor receptor expression. J Nucl Med 2006;47:2048-56.

165. Backer M V., Levashova Z, Patel V, et al. Molecular imaging of VEGF receptors in angiogenic vasculature with single-chain VEGF-based probes. Nat Med 2007;13:504-9.

166. Carlin S, Zhang H, Reese M, Ramos NN, Chen Q, Ricketts SA. A comparison of the imaging characteristics and microregional distribution of 4 hypoxia PET tracers. J Nucl Med 2014;55:515-21.

167. Kelada OJ, Carlson DJ. Molecular imaging of tumor hypoxia with positron emission tomography. Radiat Res 2014;181:335-49.

168. Kobayashi K, Hirata K, Yamaguchi S, et al. FMISO PET at 4 hours showed a better lesion-to-background ratio uptake than 2 hours in brain tumors. J Nucl Med 2015;

169. Kawai N, Lin W, Cao WD, et al. Correlation between 18F-fluoromisonidazole PET and expression of HIF-1 $\alpha$ and VEGF in newly diagnosed and recurrent malignant gliomas. Eur J Nucl Med Mol Imaging 2014;41:1870-8.

170. Gérard M, Corroyer-Dulmont A, Lesueur P, et al. Hypoxia Imaging and Adaptive Radiotherapy: A State-of-the-Art Approach in the Management of Glioma. Front Med 2019;6:117.

171. Peeters SGJA, Zegers CML, Lieuwes NG, et al. A comparative study of the hypoxia PET tracers [18F]HX4, [18F]FAZA, and [18F] 
FMISO in a preclinical tumor model. Int J Radiat Oncol Biol Phys 2015;91:351-9.

172. Segard T, Robins PD, Yusoff IF, et al. Detection of hypoxia with 18F-fluoromisonidazole ( 18F-FMISO) PET/CT in suspected or proven pancreatic cancer. Clin Nucl Med 2013;38:1-6.

173. Servagi-Vernat S, Differding S, Hanin FX, et al. A prospective clinical study of 18 F-FAZA PET-CT hypoxia imaging in head and neck squamous cell carcinoma before and during radiation therapy. Eur J Nucl Med Mol Imaging 2014;41:1544-52.

174. Tachibana I, Nishimura Y, Shibata T, et al. A prospective clinical trial of tumor hypoxia imaging with 18f-fluoromisonidazole positron emission tomography and computed tomography (f-miso pet/ct) before and during radiation therapy. $J$ Radiat Res 2013;54:1078-84.

175. Yu W, Qiao F, Su X, et al. 18F-HX4/18F-FMISO-based micro PET for imaging of tumor hypoxia and radiotherapy-associated changes in mice. Biomed Pharmacother 2019;119:109454.

176. Bourgeois M, Rajerison H, Guerard F, et al. Contribution of [64Cu]-ATSM PET in molecular imaging of tumour hypoxia compared to classical [18F] -MISO - a selected review. Nucl Med Rev Cent East Eur 2011;14:90-5..

177. Wu Y, Hao G, Ramezani S, et al. [(68)Ga]-HP-DO3A-nitroimidazole: A promising agent for PET detection of tumor hypoxia. Contrast Media Mol Imaging 2015;10:465-72.

178. Flavell RR, Truillet C, Regan MK, et al. Caged [(18)F]FDG Glycosylamines for Imaging Acidic Tumor Microenvironments Using Positron Emission Tomography. Bioconjug Chem 2016;27:170-8.

179. Wyatt LC, Lewis JS, Andreev OA, Reshetnyak YK, Engelman DM. Applications of pHLIP Technology for Cancer Imaging and Therapy. Trends Biotechnol 2017;35:653-64.

180. Vavere AL, Biddlecombe GB, Spees WM, et al. A novel technology for the imaging of acidic prostate tumors by positron emission tomography. Cancer Res 2009;69:4510-6.

181. Demoin DW, Wyatt LC, Edwards KJ, et al. PET Imaging of Extracellular pH in Tumors with 64Cu- and 18F-Labeled pHLIP Peptides: A Structure-Activity Optimization Study. Bioconjug Chem 2016;27:2014-23.

182. Hanafy NA, Dini L, Citti C, Cannazza G, Leporatti S. Inihibition of glycolysis by using a micro/nano-lipid bromopyruvic chitosan carrier as a promising tool to improve treatment of hepatocellular carcinoma. Nanomaterials 2018;8:34.

183. Miao Y, Zhang LF, Guo R, et al. (18)F-FDG PET/CT for Monitoring the Response of Breast Cancer to miR-143-Based Therapeutics by Targeting Tumor Glycolysis. Mol Ther Nucleic Acids 2016 Aug 30;5:e357.

184. Witney TH, James ML, Shen B, et al. PET imaging of tumor glycolysis downstream of hexokinase through noninvasive measurement of pyruvate kinase M2. Sci Transl Med 2015;7:310ra169.

185. Kwee SA, Lim J. Metabolic positron emission tomography imaging of cancer: Pairing lipid metabolism with glycolysis. World J Radiol 2016;8:851-6.

186. Lee N, Choi SH, Hyeon T. Nano-sized CT contrast agents. Adv Mater 2013;25:2641-60.

187. Wang CL, Cohan RH, Ellis JH, Adusumilli S, Dunnick NR. Frequency, management, and outcome of extravasation of nonionic iodinated contrast medium in 69,657 intravenous injections. Radiology 2007;243:80-7.

188. Sahani DV, Kalva SP, Hamberg LM, et al. Assessing tumor perfusion and treatment response in rectal cancer with multisection CT: initial observations. Radiology 2005;234:785-92.

189. Fushiki H, Kanoh-Azuma T, Katoh M, et al. Quantification of mouse pulmonary cancer models by microcomputed tomography imaging. Cancer Sci 2009; 100:1544-9.

190. Samei E, Saunders RS, Badea CT, et al. Micro-CT imaging of breast tumors in rodents using a liposomal, nanoparticle contrast agent. Int J Nanomedicine 2009;4:277-82.

191. Ghaghada KB, Badea CT, Karumbaiah L, et al. Evaluation of tumor microenvironment in an animal model using a nanoparticle contrast agent in computed tomography imaging. Acad Radiol 2011;18:20-30.

192. Liu Y, Ai K, Lu L. Nanoparticulate X-ray computed tomography contrast agents: From design validation to in vivo applications. Acc Chem Res 2012;45:1817-27.

193. Meng X, Zhang H, Zhang M, et al. Negative CT Contrast Agents for the Diagnosis of Malignant Osteosarcoma. Adv Sci 2019;6:1901214.

194. Meng X, Wu Y, Bu W. Functional CT Contrast Nanoagents for the Tumor Microenvironment. Adv Healthc Mater 2020;e2000912.

195. Zou Y, Wei Y, Sun Y, et al. Cyclic RGD-functionalized and disulfide-crosslinked iodine-rich polymersomes as a robust and smart theranostic agent for targeted CT imaging and chemotherapy of tumor. Theranostics 2019;9:8061-72.

196. Luo G, Yu X, Jin C, et al. LyP-1-conjugated nanoparticles for targeting drug delivery to lymphatic metastatic tumors. Int $J$ Pharm 2010;385:150-6.

197. Xing H, Zheng X, Ren Q, et al. Computed tomography imaging-guided radiotherapy by targeting upconversion nanocubes with significant imaging and radiosensitization enhancements. Sci Rep 2013;3:1751.

198. Shi H, Wang Z, Huang C, et al. A Functional CT Contrast Agent for In Vivo Imaging of Tumor Hypoxia. Small 2016;12:3995-4006.

199. Apte S, T. Chin F, E. Graves E. Molecular Imaging of Hypoxia: Strategies for Probe Design and Application. Curr Org Synth 2011;8:593603.

200. Huo D, Liu S, Zhang C, et al. Hypoxia-Targeting, Tumor Microenvironment Responsive Nanocluster Bomb for Radical-Enhanced Radiotherapy. ACS Nano 2017;11:10159-74.

201. Facciabene A, Peng X, Hagemann IS, et al. Tumour hypoxia promotes tolerance and angiogenesis via CCL28 and T reg cells. Nature 2011;475:226-30

202. Wang A, Yin L, He L, et al. An acidic pH/reduction dual-stimuli responsive nanoprobe for enhanced $\mathrm{CT}$ imaging of tumours in vivo. Nanoscale 2018;10:20126-30.

203. Xiong D, Zhang X, Peng S, Gu H, Zhang L. Smart pH-sensitive micelles based on redox degradable polymers as DOX/GNPs carriers for 
controlled drug release and CT imaging. Colloids Surf B Biointerfaces 2018;163:29-40.

204. Lusic H, Grinstaff MW. X-ray-computed tomography contrast agents. Chem Rev 2013;113:1641-66.

205. Tharkar P, Varanasi R, Wong WSF, Jin CT, Chrzanowski W. Nano-Enhanced Drug Delivery and Therapeutic Ultrasound for Cancer Treatment and Beyond. Front Bioeng Biotechnol 2019;7:324.

206. Zhou QL, Chen ZY, Wang YX, Yang F, Lin Y, Liao YY. Ultrasound-mediated local drug and gene delivery using nanocarriers. Biomed Res Int 2014;2014:963891.

207. Chowdhury SM, Lee T, Willmann JK. Ultrasound-guided drug delivery in cancer. Ultrasonography 2017;36:171-84.

208. Ghoshal G, Oelze ML, O’brien WD. Quantitative ultrasound history and successes. In: Quantitative Ultrasound in Soft Tissues 2013.

209. Feleppa EJ, Mamou J, Porter CR, MacHi J. Quantitative ultrasound in cancer imaging. Semin Oncol 2011;38:136-50.

210. Destrempes F, Cloutier G. Review of envelope statistics models for quantitative ultrasound imaging and tissue characterization. In: Quantitative Ultrasound in Soft Tissues 2013.

211. Mamou J, Oelze ML. Quantitative ultrasound in soft tissues. Quantitative Ultrasound in Soft Tissues 2013.

212. Lizzi FL, Ostromogilsky M, Feleppa EJ, Rorke MC, Yaremko MM. Relationship of Ultrasonic Spectral Parameters to Features of Tissue Microstructure. IEEE Trans Ultrason Ferroelectr Freq Control 1987;34:319-29.

213. Chandraratna PA, Whittaker P, Chandraratna PM, Gallet J, Kloner RA, Hla A. Characterization of collagen by high-frequency ultrasound: evidence for different acoustic properties based on collagen fiber morphologic characteristics. Am Heart J 1997;133:364-8.

214. Kreitz S, Dohmen G, Hasken S, Schmitz-Rode T, Mela P, Jockenhoevel S. Nondestructive method to evaluate the collagen content of fibrin-based tissue engineered structures via ultrasound. Tissue Eng Part C Methods 2011;17:1021-6.

215. Mercado KP, Helguera M, Hocking DC, Dalecki D. Noninvasive Quantitative Imaging of Collagen Microstructure in Three-Dimensional Hydrogels Using High-Frequency Ultrasound. Tissue Eng Part C Methods 2015;21:671-82.

216. Riggin CN, Sarver JJ, Freedman BR, Thomas SJ, Soslowsky LJ. Analysis of collagen organization in mouse achilles tendon using highfrequency ultrasound imaging. J Biomech Eng 2014;136:021029.

217 Mohanty K, Papadopoulou V, Newsome IG, Shelton S, Dayton PA, Muller M. Ultrasound multiple scattering with microbubbles can differentiate between tumor and healthy tissue in vivo. Phys Med Biol 2019;64:115022.

218. Gessner RC, Frederick CB, Foster FS, Dayton PA. Acoustic angiography: a new imaging modality for assessing microvasculature architecture. Int J Biomed Imaging 2013;2013:936593.

219. Dayton PA, Ferrara KW. Targeted imaging using ultrasound. J Magn Reson Imaging 2002;16:362-77.

220. Lindsey B, Rojas J, Martin K, Shelton S, Dayton P. Acoustic characterization of contrast-to-tissue ratio and axial resolution for dualfrequency contrast-specific acoustic angiography imaging. IEEE Trans Ultrason Ferroelectr Freq Control 2014;61:1668-87.

221. Shelton SE, Lee YZ, Lee M, et al. Quantification of microvascular tortuosity during tumor evolution using acoustic angiography. Ultrasound Med Biol 2015;41:1896-904.

222. Shelton SE, Lindsey BD, Tsuruta JK, Foster FS, Dayton PA. Molecular acoustic angiography: a new technique for high-resolution superharmonic ultrasound molecular imaging. Ultrasound Med Biol 2016;42:769-81.

223. Dunleavey JM, Xiao L, Thompson J, et al. Vascular channels formed by subpopulations of PECAM1+ melanoma cells. Nat Commun 2014;5:5200.

224. Kasoji SK, Rivera JN, Gessner RC, Chang SX, Dayton PA. Early assessment of tumor response to radiation therapy using high-resolution quantitative microvascular ultrasound imaging. Theranostics 2018;8:156-68.

225. Jiang Z zhen, Huang Y hua, Shen H liang, Liu X tian. Clinical applications of superb microvascular imaging in the liver, breast, thyroid, skeletal muscle, and carotid plaques. J Ultrasound Med 2019;38:2811-20.

226. Christensen-Jeffries K, Couture O, Dayton PA, et al. Super-resolution ultrasound imaging. Ultrasound Med Biol 2020;46:865-91.

227. Leung K. Microbubbles conjugated with anti-matrix metalloproteinase 2 mouse monoclonal antibody sc-13595. In: Molecular Imaging and Contrast Agent Database (MICAD) [Internet]. Bethesda (MD): National Center for Biotechnology Information (US); 2004-2013.

228. Pochon S, Tardy I, Bussat P, et al. BR55: a lipopeptide-based VEGFR2-targeted ultrasound contrast agent for molecular imaging of angiogenesis. Invest Radiol 2010;45:89-95.

229. Sugimoto K, Moriyasu F, Negishi Y, et al. Quantification in molecular ultrasound imaging a comparative study in mice between healthy liver and a human hepatocellular carcinoma xenograft. J Ultrasound Med 2012;31:1909-16.

230. Pysz MA, Foygel K, Rosenberg J, Gambhir SS, Schneider M, Willmann JK. Antiangiogenic cancer therapy: Monitoring with molecular US and a clinically translatable contrast agent (BR55). Radiology 2010;256:519-27.

231. Bzyl J, Palmowski M, Rix A, et al. The high angiogenic activity in very early breast cancer enables reliable imaging with VEGFR2targeted microbubbles (BR55). Eur Radiol 2013;23:468-75.

232. Lindsey BD, Shelton SE, Foster FS, Dayton PA. Assessment of Molecular acoustic angiography for combined microvascular and molecular imaging in preclinical tumor models. Mol Imaging Biol 2017;19:194-202.

233. Lakshman M, Needles A. Screening and quantification of the tumor microenvironment with micro-ultrasound and photoacoustic imaging. Nat Methods 2015; 12:iii-v.

234. Doyley MM, Parker KJ. Elastography: general principles and clinical applications. Ultrasound Clinics 2014;9:1-11.

235. Swartz MA, Lund AW. Lymphatic and interstitial flow in the tumour microenvironment: linking mechanobiology with immunity. Nat Rev Cancer 2012;12:210-9.

236. Itoh A, Ueno E, Tohno E, et al. Breast disease: Clinical application of US elastography for diagnosis. Radiology 2006;239:341-50.

237. Cho N, Woo KM, Jeong SP, Joo HC, Jang M, Min HS. Nonpalpable breast masses: evaluation by US elastography. Korean J Radiol 2008;9:111-8 
238. Cho N, Jang M, Lyou CY, Park JS, Choi HY, Moon WK. Distinguishing benign from malignant masses at breast US: Combined US elastography and color Doppler US-influence on radiologist accuracy. Radiology 2012;262:80-90.

239. Yi A, Cho N, Chang JM, Koo HR, La Yun B, Moon WK. Sonoelastography for 1786 non-palpable breast masses: Diagnostic value in the decision to biopsy. Eur Radiol 2012;22:1033-40.

240. Hao Y, Guo X, Ma B, Zhu L, Liu L. Relationship between ultrasound elastography and myofibroblast distribution in breast cancer and its clinical significance. Sci Rep 2016;6:19584.

241. Wang Y, Yao B, Li H, et al. Assessment of tumor stiffness with shear wave elastography in a human prostate cancer xenograft implantation model. J Ultrasound Med 2017;36:955-63.

242. Riegler J, Labyed Y, Rosenzweig S, et al. Tumor elastography and its association with collagen and the tumor microenvironment. Clin Cancer Res 2018;24:4455-67.

243. Insana MF, Pellot-Barakat C, Sridhar M, Lindfors KK. Viscoelastic imaging of breast tumor microenvironment with ultrasound. $J$ Mammary Gland Biol Neoplasia 2004;9:393-404.

244. Wang H, Mislati R, Ahmed R, et al. Elastography can map the local inverse relationship between shear modulus and drug delivery within the pancreatic ductal adenocarcinoma microenvironment. Clin Cancer Res 2019;25:2136-43.

245. Massoud TF, Gambhir SS. Molecular imaging in living subjects: Seeing fundamental biological processes in a new light. Genes Dev 2003; 17:545-80.

246. Weissleder R, Pittet MJ. Imaging in the era of molecular oncology. Nature 2008;452:580-9.

247. Weissleder R, Nahrendorf M. Advancing biomedical imaging. Proc Natl Acad Sci U S A 2015;112:14424-8.

248. Imamura T, Saitou T, Kawakami R. In vivo optical imaging of cancer cell function and tumor microenvironment. Cancer Sci 2018;109:912-8.

249. Jeevarathinam AS, Lemaster JE, Chen F, Zhao E, Jokerst J V. Photoacoustic imaging quantifies drug release from nanocarriers via redox chemistry of dye-labeled cargo. Angew Chem Int Ed Engl 2020;59:4678-83.

250. Laufer JG, Zhang EZ, Treeby BE, et al. In vivo preclinical photoacoustic imaging of tumor vasculature development and therapy. $J$ Biomed Opt 2012;17:056016.

251. Lao Y, Xing D, Yang S, Xiang L. Noninvasive photoacoustic imaging of the developing vasculature during early tumor growth. Phys Med Biol 2008;53:4203-12.

252. Omar M, Schwarz M, Soliman D, Symvoulidis P, Ntziachristos V. Pushing the optical imaging limits of cancer with multi-frequencyband raster-scan optoacoustic mesoscopy (RSOM). Neoplasia 2015;17:208-14.

253. Chekkoury A, Nunes A, Gateau J, et al. High-resolution multispectral optoacoustic tomography of the vascularization and constitutive hypoxemia of cancerous tumors. Neoplasia 2016;18:459-67.

254. Li ML, Oh JT, Xie X, et al. Simultaneous molecular and hypoxia imaging of brain tumors in vivo using spectroscopic photoacoustic tomography. Proc IEEE 2008;

255. Raes F, Sobilo J, Le Mee M, et al. High resolution ultrasound and photoacoustic imaging of orthotopic lung cancer in mice: New perspectives for onco- pharmacology. PLoS One 2016;11:e0153532.

256. Lavaud J, Henry M, Coll JL, Josserand V. Exploration of melanoma metastases in mice brains using endogenous contrast photoacoustic imaging. Int J Pharm 2017;532:704-9.

257. Imai T, Muz B, Yeh CH, et al. Direct measurement of hypoxia in a xenograft multiple myeloma model by optical-resolution photoacoustic microscopy. Cancer Biol Ther 2017;18:101-5.

258. Quiros-Gonzalez I, Tomaszewski MR, Aitken SJ, et al. Optoacoustics delineates murine breast cancer models displaying angiogenesis and vascular mimicry. Br J Cancer 2018;118:1098-106.

259. Bendinger AL, Glowa C, Peter J, Karger CP. Photoacoustic imaging to assess pixel-based sO2 distributions in experimental prostate tumors. J Biomed Opt 2018;23:1-11.

260. Tomaszewski MR, Gonzalez IQ, O’Connor JP, et al. Oxygen enhanced Optoacoustic Tomography (OE-OT) reveals vascular dynamics in murine models of prostate cancer. Theranostics 2017;7:2900-13.

261. van den Berg PJ, Daoudi K, Steenbergen W. Review of photoacoustic flow imaging: its current state and its promises. Photoacoustics 2015;3:89-99.

262. Bohndiek SE, Sasportas LS, Machtaler S, Jokerst JV, Hori S, Gambhir SS. Photoacoustic tomography detects early vessel regression and normalization during ovarian tumor response to the antiangiogenic therapy trebananib. J Nucl Med 2015;56:1942-7.

263. Rich LJ, Seshadri M. Photoacoustic monitoring of tumor and normal tissue response to radiation. Sci Rep 2016;6:21237.

264. Martinho Costa M, Shah A, Rivens I, et al. Photoacoustic imaging for the prediction and assessment of response to radiotherapy in vivo 2018;44:329516.

265. Knox HJ, Hedhli J, Kim TW, Khalili K, Dobrucki LW, Chan J. A bioreducible N-oxide-based probe for photoacoustic imaging of hypoxia. Nat Commun 2017;8:1794.

266. Chatni MR, Yao J, Danielli A, Favazza CP, Maslov KI, Wang LV. Functional photoacoustic microscopy of pH. J Biomed Opt 2011;16:100503.

267. Jo J, Lee CH, Kopelman R, Wang X. In vivo quantitative imaging of tumor $\mathrm{pH}$ by nanosonophore assisted multispectral photoacoustic imaging. Nat Commun 2017;8:471.

268. Lei H, Johnson LA, Liu S, et al. Characterizing intestinal inflammation and fibrosis in Crohn's disease by photoacoustic imaging: feasibility study. Biomed Opt Express 2016;7:2837-48.

269. Zhu Y, Johnson LA, Huang Z, et al. Identifying intestinal fibrosis and inflammation by spectroscopic photoacoustic imaging: an animal 
study in vivo. Biomed Opt Express 2018;9:1590-1600.

270. Cao Y, Kole A, Hui J, et al. Fast assessment of lipid content in arteries in vivo by intravascular photoacoustic tomography. Sci Rep 2018;8:2400.

271. Wu PC, Hsieh TY, Tsai ZU, Liu TM. In vivo quantification of the structural changes of collagens in a melanoma microenvironment with second and third harmonic generation microscopy. Sci Rep 2015;5:8879.

272. Dragulescu-Andrasi A, Kothapalli SR, Tikhomirov GA, Rao J, Gambhir SS. Activatable oligomerizable imaging agents for photoacoustic imaging of furin-like activity in living subjects. J Am Chem Soc 2013;135:11015-22.

273. Levi J, Kothapalli SR, Bohndiek S, et al. Molecular photoacoustic imaging of follicular thyroid carcinoma. Clin Cancer Res 2013;19:1494-502.

274. Yin L, Sun H, Zhang H, et al. Quantitatively visualizing tumor-related protease activity in vivo using a ratiometric photoacoustic probe. $J$ Am Chem Soc 2019;141:3265-73.

275. Egeblad M, Werb Z. New functions for the matrix metalloproteinases in cancer progression. Nat Rev Cancer 2002;2:161-74.

276. Tzoumas S, Zaremba A, Klemm U, Nunes A, Schaefer K, Ntziachristos V. Immune cell imaging using multi-spectral optoacoustic tomography. Opt Lett 2014;39:3523-6.

277. Zheng S, Li H, Lai K, et al. Noninvasive photoacoustic and fluorescent tracking of optical dye labeled T cellular activities of diseased sites at new depth. J Biophotonics 2018;11:e201800073.

278. Filippi M, Garello F, Pasquino C, et al. Indocyanine green labeling for optical and photoacoustic imaging of mesenchymal stem cells after in vivo transplantation. J Biophotonics 2019;12:e201800035.

279. Weber J, Beard PC, Bohndiek SE. Contrast agents for molecular photoacoustic imaging. Nat Methods 2016;13:639-50.

280. Burke K, Brown E. The use of second harmonic generation to image the extracellular matrix during tumor progression. Intravital 2014;3:e984509.

281. Masedunskas A, Milberg O, Porat-Shliom N, et al. Intravital microscopy: a practical guide on imaging intracellular structures in live animals. Bioarchitecture 2012;2:143-57.

282. Sturm MB, Joshi BP, Lu S, et al. Targeted imaging of esophageal neoplasia with a fluorescently labeled peptide: first-in-human results. Sci Transl Med 2013;5:184ra61.

283. Pan Y, Volkmer JP, Mach KE, et al. Endoscopic molecular imaging of human bladder cancer using a CD47 antibody. Sci Transl Med 2014;6:260ra148.

284. Ricard C, Stanchi F, Rodriguez T, Amoureux MC, Rougon G, Debarbieux F. Dynamic quantitative intravital imaging of glioblastoma progression reveals a lack of correlation between tumor growth and blood vessel density. PLoS One 2013;8:e72655.

285. Fisher DT, Muhitch JB, Kim M, et al. Intraoperative intravital microscopy permits the study of human tumour vessels. Nat Commun 2016;7:10684.

286. Zomer A, Maynard C, Verweij FJ, et al. In vivo imaging reveals extracellular vesicle-mediated phenocopying of metastatic behavior. Cell 2015;161:1046-57.

287. Qi S, Li H, Lu L, et al. Long-term intravital imaging of the multicolor-coded tumor microenvironment during combination immunotherapy. Elife 2016;5:e14756.

288. Arlauckas SP, Garris CS, Kohler RH, et al. In vivo imaging reveals a tumor-associated macrophage-mediated resistance pathway in antiPD-1 therapy. Sci Transl Med 2017;9:eaal3604.

289. Karagiannis GS, Pastoriza JM, Wang Y, et al. Neoadjuvant chemotherapy induces breast cancer metastasis through a TMEM-mediated mechanism. Sci Transl Med 2017;9:eaan0026.

290. Ntziachristos V. Going deeper than microscopy: the optical imaging frontier in biology. Nat Methods 2010;7:603-14.

291. Vakoc BJ, Lanning RM, Tyrrell JA, et al. Three-dimensional microscopy of the tumor microenvironment in vivo using optical frequency domain imaging. Nat Med 2009;15:1219-23.

292. Junankar S, Shay G, Jurczyluk J, et al. Real-time intravital imaging establishes tumor-associated macrophages as the extraskeletal target of bisphosphonate action in cancer. Cancer Discov 2015;5:35-42.

293. Smith BR, Kempen P, Bouley D, et al. Shape matters: intravital microscopy reveals surprising geometrical dependence for nanoparticles in tumor models of extravasation. Nano Lett 2012;12:3369-77.

294. Winfree S, Hato T, Day RN. Intravital microscopy of biosensor activities and intrinsic metabolic states. Methods 2017;128:95-104.

295. Niesner R, Andresen V, Neumann J, Spiecker H, Gunzer M. The power of single and multibeam two-photon microscopy for highresolution and high-speed deep tissue and intravital imaging. Biophys J 2007;93:2519-29.

296. You S, Tu H, Chaney EJ, et al. Intravital imaging by simultaneous label-free autofluorescence-multiharmonic microscopy. Nat Commun 2018;9:2125.

297. Katsuno Y, Hanyu A, Kanda H, et al. Bone morphogenetic protein signaling enhances invasion and bone metastasis of breast cancer cells through Smad pathway. Oncogene 2008;27:6322-33.

298. Hara-Miyauchi C, Tsuji O, Hanyu A, et al. Bioluminescent system for dynamic imaging of cell and animal behavior. Biochem Biophys Res Commun 2012;419:188-93.

299. Rathbun CM, Prescher JA. Bioluminescent probes for imaging biology beyond the culture dish. Biochemistry 2017;56:5178-84.

300. Kim YP, Daniel WL, Xia Z, Xie H, Mirkin CA, Rao J. Bioluminescent nanosensors for protease detection based upon gold nanoparticleluciferase conjugates. Chem Commun 2010;46:76-8.

301. Xia Z, Xing Y, So MK, Koh AL, Sinclair R, Rao J. Multiplex detection of protease activity with quantum dot nanosensors prepared by intein-mediated specific bioconjugation. Anal Chem 2008;80:8649-55. 
302. Yao H, Zhang Y, Xiao F, Xia Z, Rao J. Quantum dot/bioluminescence resonance energy transfer based highly sensitive detection of proteases. Angew Chem Int Ed Engl 2007;46:4346-9.

303. Xia Z, Xing Y, Jeon J, et al. Immobilizing reporters for molecular imaging of the extracellular microenvironment in living animals. ACS Chem Biol 2011;6:1117-26.

304. Kidd S, Spaeth E, Dembinski JL, et al. Direct evidence of mesenchymal stem cell tropism for tumor and wounding microenvironments using in vivo bioluminescent imaging. Stem Cells 2009;27:2614-23.

305. Doucette T, Rao G, Yang Y, et al. Mesenchymal stem cells display tumor-specific tropism in an RCAS/Ntv-a glioma model. Neoplasia 2011;13:716-25.

306. Cao J, Hou S, Ding H, et al. In vivo tracking of systemically administered allogeneic bone marrow mesenchymal stem cells in normal rats through bioluminescence imaging. Stem Cells Int 2016;2016:3970942.

307. Allen AB, Gazit Z, Su S, Stevens HY, Guldberg RE. In vivo bioluminescent tracking of mesenchymal stem cells within large hydrogel constructs. Tissue Eng Part C Methods 2014;20:806-16.

308. Conway M, Xu T, Kirkpatrick A, Ripp S, Sayler G, Close D. Real-time tracking of stem cell viability, proliferation, and differentiation with autonomous bioluminescence imaging. BMC Biol 2020;18:79.

309. Vilela CAP, Souza LEB, Siqueira RC, Calado RT, Covas DT, Paula JS. Ex vivo evaluation of intravitreal mesenchymal stromal cell viability using bioluminescence imaging. Stem Cell Res Ther 2018;9:155.

310. Baklaushev VP, Kilpeläinen A, Petkov S, et al. Luciferase expression allows bioluminescence imaging but imposes limitations on the orthotopic mouse (4T1) model of breast cancer. Sci Rep 2017;7:7715.

311. Moriyama EH, Niedre MJ, Jarvi MT, et al. The influence of hypoxia on bioluminescence in luciferase-transfected gliosarcoma tumor cells in vitro. Photochem Photobiol Sci 2008;7:675-80.

312. Khalil AA, Jameson MJ, Broaddus WC, et al. The influence of hypoxia and $\mathrm{pH}$ on bioluminescence imaging of luciferase-transfected tumor cells and xenografts. Int J Mol Imaging 2013;2013:287697.

313. Gao Y, Lin Y, Liu T, et al. Bioluminescent probe for tumor hypoxia detection via CYP450 reductase in living animals. Anal Chem 2017;89:12488-93.

314. Saha D, Dunn H, Zhou H, et al. In vivo bioluminescence imaging of tumor hypoxia dynamics of breast cancer brain metastasis in a mouse model. J Vis Exp 2011;:3175.

315. McMillin DW, Delmore J, Weisberg E, et al. Tumor cell-specific bioluminescence platform to identify stroma-induced changes to anticancer drug activity. Nat Med 2010;16:483-9.

316. Correa de Sampaio P, Auslaender D, Krubasik D, et al. A heterogeneous in vitro three dimensional model of tumour-stroma interactions regulating sprouting angiogenesis. PLoS One 2012;7:e30753.

317. Hoffman RM. The multiple uses of fluorescent proteins to visualize cancer in vivo. Nat Rev Cancer 2005;5:796-806.

318. Hoffman RM, Yang M. Subcellular imaging in the live mouse. Nat Protoc 2006;1:775-82.

319. Nakamura M, Suetsugu A, Hasegawa K, et al. Color-coded imaging distinguishes cancer cells, stromal cells, and recombinant cancerstromal cells in the tumor microenvironment during metastasis. Anticancer Res 2018;38:4417-23.

320. Yang M, Li L, Jiang P, Moossa AR, Penman S, Hoffman RM. Dual-color fluorescence imaging distinguishes tumor cells from induced host angiogenic vessels and stromal cells. Proc Natl Acad Sci U S A 2003;100:14259-62.

321. Yang M, Jiang P, Hoffman RM. Whole-body subcellular multicolor imaging of tumor-host interaction and drug response in real time. Cancer Res 2007;67:5195-200.

322. Egeblad M, Ewald AJ, Askautrud HA, et al. Visualizing stromal cell dynamics in different tumor microenvironments by spinning disk confocal microscopy. Dis Model Mech 2008;1:155-67; discussion 165.

323. Mochizuki N, Yamashita S, Kurokawa K, et al. Spatio-temporal images of growth-factor-induced activation of Ras and Rap1. Nature 2001;411:1065-8.

324. Brown LF, Berse B, Jackman RW, et al. Expression of vascular permeability factor (vascular endothelial growth factor) and its receptors in breast cancer. Hum Pathol 1995;26:86-91.

325. Hanyu A, Kojima K, Hatake K, et al. Functional in vivo optical imaging of tumor angiogenesis, growth, and metastasis prevented by administration of anti-human VEGF antibody in xenograft model of human fibrosarcoma HT1080 cells. Cancer Sci 2009;100:2085-92.

326. Mayes PA, Dicker DT, Liu YY, El-Deiry WS. Noninvasive vascular imaging in fluorescent tumors using multispectral unmixing. Biotechniques 2008;45:459-60, 461-4.

327. Li G, Xing Y, Wang J, Conti PS, Chen K. Near-infrared fluorescence imaging of CD13 receptor expression using a novel Cy5.5-labeled dimeric NGR peptide. Amino Acids 2014;46:1547-56.

328. Biswal NC, Gamelin JK, Yuan B, Backer MV, Backer JM, Zhu Q. Fluorescence imaging of vascular endothelial growth factor in tumors for mice embedded in a turbid medium. J Biomed Opt 2010;15:016012.

329. Choi M, Chung T, Choi K, Choi C. Dynamic fluorescence imaging for multiparametric measurement of tumor vasculature. In: Imaging, Manipulation, and Analysis of Biomolecules, Cells, and Tissues VII. 2009.

330. Zhang Y, So MK, Rao J. Protease-modulated cellular uptake of quantum dots. Nano Lett 2006;6:1988-92.

331. Xie J, Zhang F, Aronova M, et al. Manipulating the power of an additional phase: a flower-like au-Fe $3 \mathrm{O} 4$ optical nanosensor for imaging protease expressions in vivo. ACS Nano 2011;5:3043-51.

332. Cobos-Correa A, Trojanek JB, Diemer S, Mall MA, Schultz C. Membrane-bound FRET probe visualizes MMP12 activity in pulmonary inflammation. Nat Chem Biol 2009;5:628-30.

333. Park SY, Lee SM, Kim GB, Kim YP. Gold nanoparticle-based fluorescence quenching via metal coordination for assaying protease 
activity. Gold Bull 2012;45:213-9.

334. Lee S, Cha EJ, Park K, et al. A near-infrared-fluorescence-quenched gold-nanoparticle imaging probe for in vivo drug screening and protease activity determination. Angew Chem Int Ed Engl 2008;47:2804-7

335. Li X, Shepard HM, Cowell JA, et al. Parallel accumulation of tumor hyaluronan, collagen, and other drivers of tumor progression. Clin Cancer Res 2018;24:4798-807.

336. Qi B, Crawford AJ, Wojtynek NE, et al. Tuned near infrared fluorescent hyaluronic acid conjugates for delivery to pancreatic cancer for intraoperative imaging. Theranostics 2020;10:3413-29.

337. Lopès A, Cassé AH, Billard E, et al. Deciphering the immune microenvironment of a tissue by digital imaging and cognition network. Sci Rep 2018;8:16692.

338. Ke CC, Liu RS, Suetsugu A, et al. In vivo fluorescence imaging reveals the promotion of mammary tumorigenesis by mesenchymal stromal cells. PLoS One 2013;8:e69658.

339. Germain RN, Miller MJ, Dustin ML, Nussenzweig MC. Dynamic imaging of the immune system: progress, pitfalls and promise. Nat Rev Immunol 2006 Jul;6:497-507.

340. Quante M, Tu SP, Tomita H, et al. Bone marrow-derived myofibroblasts contribute to the mesenchymal stem cell niche and promote tumor growth. Cancer Cell 2011;19:257-72.

341. Zhao H, Yang L, Baddour J, et al. Tumor microenvironment derived exosomes pleiotropically modulate cancer cell metabolism. Elife 2016;5:e10250.

342. Li YY, Tao YW, Gao S, et al. Cancer-associated fibroblasts contribute to oral cancer cells proliferation and metastasis via exosomemediated paracrine miR-34a-5p. EBioMedicine 2018;36:209-20.

343. Lo PC, Chen J, Stefflova K, et al. Photodynamic molecular beacon triggered by fibroblast activation protein on cancer-associated fibroblasts for diagnosis and treatment of epithelial cancers. J Med Chem 2009;52:358-68.

344. Cai Q, Yu T, Zhu W, Xu Y, Qian X. A turn-on fluorescent probe for tumor hypoxia imaging in living cells. Chem Commun 2015;51:1473941.

345. Tian X, Li Z, Sun Y, Wang P, Ma H. Near-infrared fluorescent probes for hypoxia detection via joint regulated enzymes: design, synthesis, and application in living cells and mice. Anal Chem 2018;90:13759-66.

346. Yang D, Tian HY, Zang TN, Li M, Zhou Y, Zhang JF. Hypoxia imaging in cells and tumor tissues using a highly selective fluorescent nitroreductase probe. Sci Rep 2017;7:9174.

347. Mirabello V, Cortezon-Tamarit F, Pascu SI. Oxygen sensing, hypoxia tracing and in vivo imaging with functional metalloprobes for the early detection of non-communicable diseases. Front Chem 2018;6:27.

348. Wang L, Li C. PH responsive fluorescence nanoprobe imaging of tumors by sensing the acidic microenvironment. J Mater Chem 2011;21:15862-71.

349. Fang M, Adhikari R, Bi J, et al. Fluorescent probes for sensitive and selective detection of $\mathrm{pH}$ changes in live cells in visible and nearinfrared channels. J Mater Chem B 2017;5:9579-90.

350. Liu Q, Zheng C, Zhao H, Wang K, Tao W. Tumor cell pH detection based on CdSe quantum dots' fluorescence charateristics. Technol Health Care 2019;27:239-47.

351. Anemone A, Consolino L, Arena F, Capozza M, Longo DL. Imaging tumor acidosis: a survey of the available techniques for mapping in vivo tumor pH. Cancer Metastasis Rev 2019;38:25-49.

352. Stuker F, Ripoll J, Rudin M. Fluorescence molecular tomography: principles and potential for pharmaceutical research. Pharmaceutics 2011;3:229-74.

353. Leimgruber A, Berger C, Cortez-Retamozo V, et al. Behavior of endogenous Tumor-associated macrophages assessed in vivo using a functionalized nanoparticle. Neoplasia 2009;11:459-68, 2 p following 468.

354. Li L, Du Y, Chen X, Tian J. Fluorescence molecular imaging and tomography of matrix metalloproteinase-activatable near-infrared fluorescence probe and image-guided orthotopic glioma resection. Mol Imaging Biol 2018;20:930-9.

355. Zhang Q, Bindokas V, Shen J, Fan H, Hoffman RM, Xing HR. Time-course imaging of therapeutic functional tumor vascular normalization by antiangiogenic agents. Mol Cancer Ther 2011;10:1173-84.

356. Montet X, Figueiredo JL, Alencar H, Ntziachristos V, Mahmood U, Weissleder R. Tomographic fluorescence imaging of tumor vascular volume in mice. Radiology 2007;242:751-8.

357. Theek B, Gremse F, Kunjachan S, et al. Characterizing EPR-mediated passive drug targeting using contrast-enhanced functional ultrasound imaging. J Control Release 2014;182:83-9.

358. Ntziachristos V, Tung CH, Bremer C, Weissleder R. Fluorescence molecular tomography resolves protease activity in vivo. Nat Med 2002;8:757-60.

359. Fujimoto JG, Pitris C, Boppart SA, Brezinski ME. Optical coherence tomography: an emerging technology for biomedical imaging and optical biopsy. Neoplasia 2000;2:9-25.

360. Goswami M, Wang X, Zhang P, et al. Novel window for cancer nanotheranostics: non-invasive ocular assessments of tumor growth and nanotherapeutic treatment efficacy in vivo. Biomed Opt Express 2019;10:151-66.

361. Marvdashti T, Duan L, Aasi SZ, Tang JY, Ellerbee Bowden AK. Classification of basal cell carcinoma in human skin using machine learning and quantitative features captured by polarization sensitive optical coherence tomography. Biomed Opt Express 2016;7:3721-35.

362. Kiseleva E, Kirillin M, Feldchtein F, et al. Differential diagnosis of human bladder mucosa pathologies in vivo with cross-polarization optical coherence tomography. Biomed Opt Express 2015;6:1464-76.

363. Chhetri RK, Phillips ZF, Troester MA, Oldenburg AL. Longitudinal study of mammary epithelial and fibroblast co-cultures using optical 
coherence tomography reveals morphological hallmarks of pre-malignancy. PLoS One 2012;2012;7:e49148.

364. Winkler AM, Rice PFS, Weichsel J, et al. In vivo, dual-modality OCT/LIF imaging using a novel VEGF receptor-targeted NIR fluorescent probe in the AOM-treated mouse model. Mol Imaging Biol 2011;13:1173-82.

365. De Carvalho N, Welzel J, Schuh S, et al. The vascular morphology of melanoma is related to Breslow index: an in vivo study with dynamic optical coherence tomography. Exp Dermatol 2018;27:1280-6.

366. Smith BR, Gambhir SS. Nanomaterials for in vivo imaging. Chem Rev 2017;117:901-86.

367. Liba O, Sorelle ED, Sen D, De La Zerda A. Contrast-enhanced optical coherence tomography with picomolar sensitivity for functional in vivo imaging. Sci Rep 2016;6:23337.

368. Si P, Yuan E, Liba O, et al. Gold nanoprisms as optical coherence tomography contrast agents in the second near-infrared window for enhanced angiography in live animals. ACS Nano 2018;12:11986-94.

369. Si P, Sen D, Dutta R, et al. In vivo molecular optical coherence tomography of lymphatic vessel endothelial hyaluronan receptors. Sci Rep 2017;7:1086.

370. Sorelle ED, Yecies DW, Liba O, et al. Spatiotemporal tracking of brain-tumor-associated myeloid cells in vivo through optical coherence tomography with plasmonic labeling and speckle modulation. ACS Nano 2019;13:7985-95.

371. John R, Rezaeipoor R, Adie SG, et al. In vivo magnetomotive optical molecular imaging using targeted magnetic nanoprobes. Proc Natl Acad Sci U S A 2010;107:8085-90.

372. Fernandes C, Suares D, Yergeri MC. Tumor Microenvironment Targeted Nanotherapy. Front Pharmacol 2018;9:1230.

373. García-Figueiras R, Baleato-González S, Padhani AR, et al. How clinical imaging can assess cancer biology. Insights Imaging 2019;10:28.

374. Wu Y, Zhang W, Li J, Zhang Y. Optical imaging of tumor microenvironment. Am J Nucl Med Mol Imaging 2013;3:1-15.

375. Youn H, Hong KJ. In vivo non invasive molecular imaging for immune cell tracking in small animals. Immune Netw 2012;12:223-9.

376. Condeelis J, Weissleder R. In vivo imaging in cancer. Cold Spring Harb Perspect Biol 2010;2:a003848.

377. Schinkel AFL, Kaspar M, Staub D. Contrast-enhanced ultrasound: clinical applications in patients with atherosclerosis. Int J Cardiovasc Imaging 2016;32:35-48.

378. Barr RG. Contrast enhanced ultrasound for focal liver lesions: how accurate is it? Abdom Radiol 2018;43:1128-33.

379. Mallidi S, Luke GP, Emelianov S. Photoacoustic imaging in cancer detection, diagnosis, and treatment guidance. Trends Biotechnol 2011;29:213-21.

380. Choo YW, Jeong J, Jung K. Recent advances in intravital microscopy for investigation of dynamic cellular behavior in vivo. $B M B$ Rep 2020;53:357-66

381. Ramamonjisoa N, Ackerstaff E. Characterization of the tumor microenvironment and tumor-stroma interaction by non-invasive preclinical imaging. Front Oncol 2017;7:3.

382. Ottobrini L, Martelli C, Trabattoni DL, Clerici M, Lucignani G. In vivo imaging of immune cell trafficking in cancer. Eur J Nucl Med Mol Imaging 2011;38:949-68.

383. Narunsky L, Oren R, Bochner F, Neeman M. Imaging aspects of the tumor stroma with therapeutic implications. Pharmacol Ther 2014;141:192-208.

384. Krohn KA, Link JM, Mason RP. Molecular imaging of hypoxia. J Nucl Med 2008;49 Suppl 2:129S-48S.

385. Dogra P, Adolphi NL, Wang Z, et al. Establishing the effects of mesoporous silica nanoparticle properties on in vivo disposition using imaging-based pharmacokinetics. Nat Commun 2018;9:4551.

386. Schmid D, Park CG, Hartl CA, et al. T cell-targeting nanoparticles focus delivery of immunotherapy to improve antitumor immunity. Nat Commun 2017;8:1747.

387. Mattheolabakis G, Mikelis CM. Nanoparticle delivery and tumor vascular normalization: the chicken or the egg? Front Oncol 2019;9:1227. 